\title{
陆域蒸散的测算理论方法: 回顾与展望
}

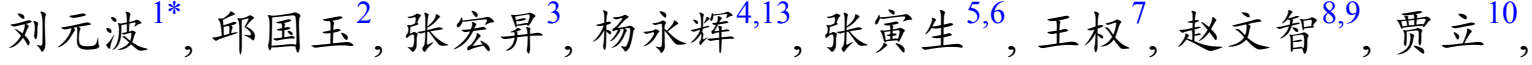 \\ 吉喜斌 8,9 , 熊育久 ${ }^{11}$, 滒春华 ${ }^{2}$, 马 宁 $^{12}$, 韩淑敏 ${ }^{4}$, 崔逸凡 ${ }^{1,13}$
}

1. 中国科学院南京地理与湖泊研究所, 南京 210008 ;

2. 北京大学深圳研究生院环境与能源学院, 深圳 518055 ;

3. 北京大学物理学院大气与海洋科学系气候与海-气实验室, 北京 100871 ;

4. 中国科学院遗传与发育生物学研究所农业资源研究中心, 中国科学院农业水资源重点实验室, 石家庄 050022;

5. 中国科学院青藏高原研究所青藏高原环境变化与地表过程重点实验室, 北京 100101;

6. 中国科学院青藏高原地球科学卓越创新中心, 北京 100101 ;

7. Faculty of Agriculture, Shizuoka University, Shizuoka 422-8529, Japan;

8. 中国科学院西北生态环境资源研究院, 兰州 730000;

9. 中国科学院内陆河流域生态水文重点实验室, 兰州 730000;

10. 中国科学院空天信息创新研究院, 北京 100094 ;

11. 中山大学土木工程学院, 广州 510275 ;

12. 中国科学院地理科学与资源研究所陆地水循环及地表过程重点实验室, 北京 100101 ;

13. 中国科学院大学, 北京 100049

* 通讯作者, E-mail: ybliu@niglas.ac.cn

收稿日期: 2020-12-01; 收修改稿日期: 2021-08-03; 接受日期: 2021-08-24; 网络版发表日期: 2021-12-01

国家自然科学基金项目(批准号: 51879255, 41430855)资助

摘要 蒸散是地球水分循环与能量转换的关键环节. 陆域蒸散的精准测算是地球物理、生物乃至环境过程研究 的共同科学难题. 以英国Dalton和Penman等人为代表的开创性工作，以及大气边界层湍流交换理论与实验的不断 发展，奠基了现代主流的蒸散测算方法. 20 世纪 90 年代以来技术趋于成熟的涡动相关系统和卫星遥感等观测手 段，跨越寒带到热带、干旱区到湿润地区，涵盖水体、湿地、森林、农田、草地、裸地、城市等不同下垫面类 型，极大地拓展了对蒸散过程认知的深度和广度; 捕捉了诸如地表夜间蒸散、蒸散迟滞现象、非均匀下垫面的 湍流间歇、平流关联的岛屿效应、下垫面转㧖效应等新的现象和事实，对经典相似性理论和蒸散测算理论等提 出了新的挑战; 最大熵增蒸散模型和非参数化蒸散模型等新方法和新理论的萌芽已经出现. 与此同时, 点位高频 观测和航空航天遥感技术构筑了从植物气孔到叶片、植株、冠层、景观、流域等跨尺度测算手段，在地表蒸散 过程观测及机理解析上，形成纵向深化及横向综合的新进展. 陆域蒸散新理念、新模型、新技术的综合性发展， 成为突破蒸散精准测算难题的基础条件，这将为揭示地球系统的水-热-碳循环机理等全球性重大基础理论问题， 为满足农业灌溉与粮食安全、水资源精细化管理与生态环境保护、城市热环境调控与全球升温适应对策等国家 重大需求, 提供更为严谨坚实的科学理论依据和实验支撑.

\footnotetext{
中文引用格式: 刘元波, 邱国玉, 张宏昇, 杨永辉, 张寅生, 王权, 赵文智, 贾立, 吉喜斌, 熊育久, 滒春华, 马宁, 韩淑敏, 崔逸凡. 2022. 陆域蒸散的测算理论方法: 回顾与展望. 中国科学: 地球科学, 52(3): 381-399, doi: 10.1360/SSTe-2020-0342

英文引用格式: Liu Y, Qiu G, Zhang H, Yang Y, Zhang Y, Wang Q, Zhao W, Jia L, Ji X, Xiong Y, Yan C, Ma N, Han S, Cui Y. 2022. Shifting from homogeneous to heterogeneous surfaces in estimating terrestrial evapotranspiration: Review and perspectives. Science China Earth Sciences, 65(2): 197-214, https:/ doi.org/10.1007/s11430-020-9834-y
} 
关键词蒸散, 大气边界层, 非均匀下垫面, 不确定性

\section{1 引言}

自然界的蒸散(evapotranspiration)是指水分子从 液态变成气态的过程. 它既包括来自土壤、水域、冠 层表面等各类地表的蒸发(evaporation), 也包括通过植 物气孔的蒸腾(transpiration)(Burman和Pochop, 1994). 液态水的比热容很大, 是同等体积空气的 3500 倍, 汽 化相变潜热很高. 因此, 伴随蒸散过程的潜热(latent heat)是地表能量收支不能忽略的重要项(Brutsaert, 1982).

在全球尺度上, 到达地面 $48 \%$ 的太阳净辐射被蒸 散过程消耗(Peixoto, 1995). 地球表面64\%的陆地降水 以蒸散方式重新进入大气, 参与地球水循环(Oki和Kanae, 2006), 干旱区的这一比例可高达90\%(Novák, 2012). 几乎所有的干旱、热浪等极端水文气象事件, 都与蒸散过程密不可分 (朴世龙等, 2019). 伴随长期地 质地貌演化的风化过程, 地球上各类地表的水热分配 过程, 湖泊、水库和农田的蒸发耗水过程, 各类气候 区的流域水量收支过程, 不同地域的土壤盐碱物理性 质, 局地干湿气团的动力和热力性质变化, 小到生物 和人体的皮表生理调节及新陈代谢过程, 大到自然生 态系统的结构与功能演替, 以及城镇乡村等人类宜居 环境建设与格局变迁, 几乎所有的地表物理要素及其 变化过程, 都受不同程度蒸散作用的影响(Fisher等, 2017).

陆域蒸散的水热耦合特性及其广泛存在性(苏涛 和封国林, 2015), 对于与日常生活密切相关的天气预 报、极端水文气候灾变事件的预测预警、长期气候趋 势的模拟预判、水库兴利的合理调度、农田灌溉的节 水提升、精准农业的高效实施、林业结构的优化种 植、草原牧业的永续经营、产业耗水的协调分配、生 活用水的定额调整、湖泊范围的扩张萎缩、荒漠绿洲 的维护保持等, 具有重要的实践价值. 在全球变温和人 类活动加剧, 人类可持续发展需求与生态环境保护意 识日益增强，践行“山水林田湖草生命共同体”的系统 思想等这一时代背景下, 加强并深化陆域蒸散研究, 具有重大的科学及实践意义(孙岗和吴国雄, 2001).
陆域蒸散涉及下垫面的复杂性及不同时空尺度测 算的不确定性, 已成为地球物理、生物和环境过程研 究的共同科学难题. 人们相继提出了实际蒸散、潜在 蒸发、平衡态蒸发、水面蒸散、农田蒸散等可测量或 不可测量的概念或物理量; 基于不同的假设条件, 发展 了多种测算公式或方法, 并衍生出多种模型或方法, 并 在相关领域得到了不同程度的应用(Brutsaert, 1982, 2005). 本文注重勾勒实际蒸散的测算理论方法的发展 脉络主线, 从长期的理论方法研究和应用案例中, 努力 甄选开创性或原创性工作, 不追求逐例归纳、面面俱 到; 力求站在学科发展与历史条件背景下, 追根溯源, 梳理不同理论方法的基本假设及局限性，以期明晰当 前理论方法存在的瓶颈, 并展望未来可能突破的路径.

\section{2 现代蒸散测算理论方法及发展历程}

有文字记载的蒸散概念, 始于公元前 8 世纪古希腊 诗人赫西俄德(Hesiod). 中国《黄帝内经・素问》中有 “地气上为云”的记载, 可视为朴素的蒸散认识. 19 世 纪初, 英国化学家Dalton(1802)提出了水面蒸发与环境 要素之间的关系, 被视为最早的定量化研究. 20 世纪中 期, 以Penman(1948)等人为代表的开创性工作, 以及大 气边界层湍流交换理论与实验的不断发展, 奠基了现 代主流的蒸散测算理论方法(Brutsaert，1982，2005) (图1).

\section{1 理论基础及方法}

在太阳辐射等因素驱动下, 地-气相互作用构成地 表蒸散过程发生发展的宏观背景条件. 地表蒸散涉及 多种物理过程, 有着十分复杂的微观驱动机制, 包括 大气动力作用、地表能量转换、液气相态转变、水气 散逸迁移以及分子吸附作用等. 自然界的蒸散作为一 种界面相变现象, 在垂直方向上, 大气状态是蒸散过 程的上边界条件, 土壤或植被等则构成下边界条件, 涉及大气边界层、地表覆被状况及水分状态变化等多 个过程, 分别属于边界层气象学、水文气象学、陆地 水文学等学科的核心内容. 不同学科从不同角度, 发 


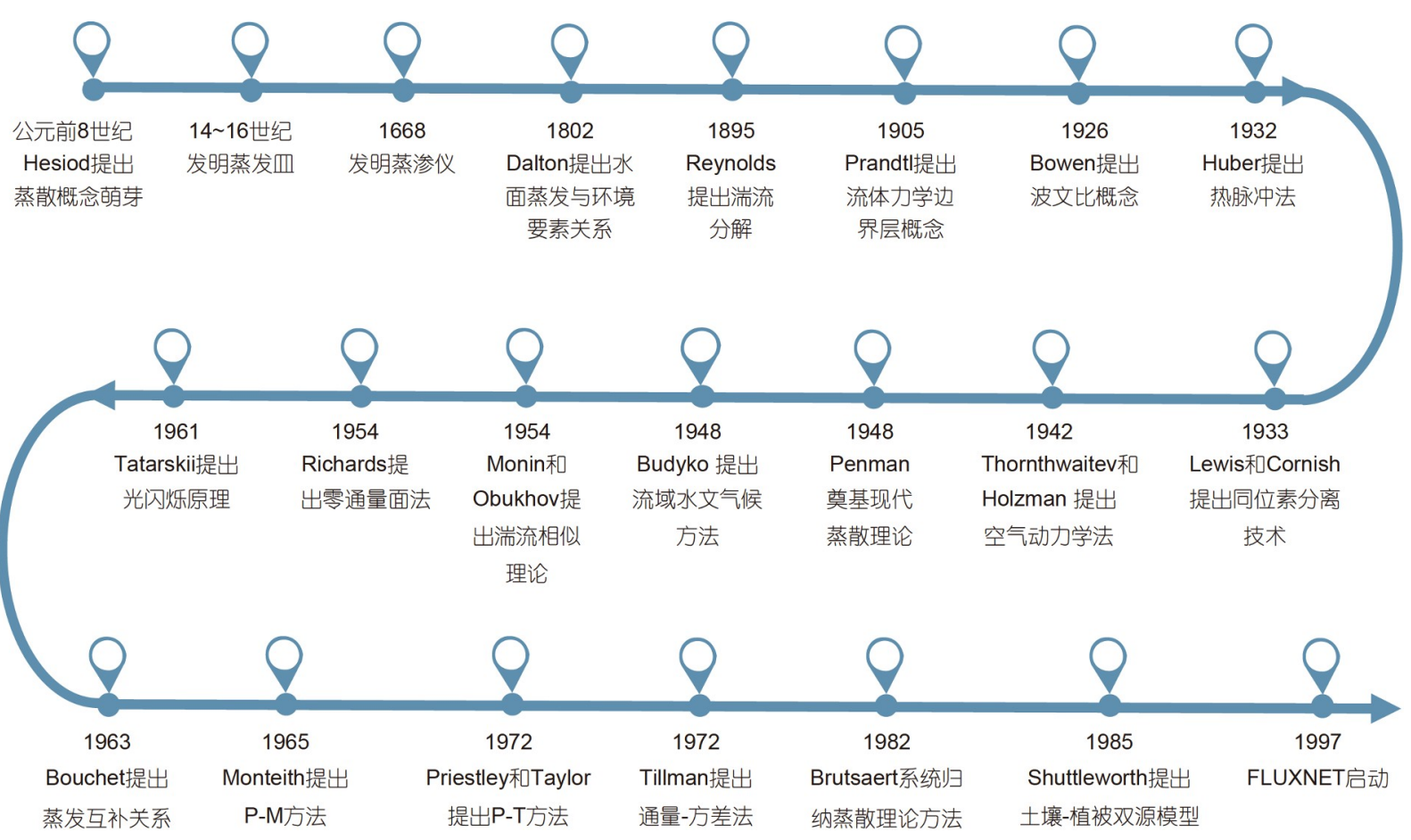

图 1 (网络版彩图)现代蒸散测算理论方法的发展历程简图

展了不同的蒸散测算理论方法. 下面结合提出时间的 先后, 分别予以概述(表1).

\subsection{1 边界层气象学原理及方法}

边界层气象学(boundary layer meteorology)源于 20 世纪初德国学者Prandtl(1905)提出的流体力学边界层 概念，以湍流(turbulence)理论和概率统计分析为基础, 包括雷诺分解(Reynolds，1895)、泰勒冻结假设(Taylor，1938)、Monin-Obukhov相似性理论(Monin和Obukhov，1954)等核心内容, 研究大气边界层(atmospheric boundary layer)结构及变化规律(Lemone等, 2018). 大 气边界层是对流层中最靠近地表的气层，垂直厚度一 般为 $1 \sim 3 \mathrm{~km}$, 存在着多种尺度的湍流运动, 蒸散研究更 关注距离地面数十米高度的低层大气. 湍流作用输送 动量、热量和物质, 使得大气边界层成为地-气间水 热、能量和能量交换与输送的重要气层. 气象要素显 著的日变化性质对陆面蒸散过程至关重要, 反之蒸散 过程也影响大气边界层的状态. 目前估算地表蒸散的 主要方法包括波文比能量平衡法、空气动力学法以及 浴动相关法等.

（1）波文比(Bowen-ratio)能量平衡法. 美国物理学
家Bowen(1926)提出了采用显热通量与潜热通量之比 (波文比), 描述水分蒸散过程中的能量分配. 波文比与 两个不同高度的温度差与湿度差的比值成正比，结合 地表的可利用能量值, 推算潜热通量(蒸散量)(Tanner, 1960；Todd等，2000). 波文比能量平衡法的物理基础 明确, 所需输入变量为常规气象观测项目, 容易推广应 用，但波文比的测算精度取决于温度差和湿度差观测 的准确性, 要求温度和湿度梯度分布明显以及下垫面 均匀且无平流的影响, 使用具有较强的限制条件. 在 温度梯度为零时，误差会无限放大(Lewis，1995; Todd 等，2000)。实际应用中务必注意该方法的使用范围 (Abtew和Melesse, 2013).

(2) 空气动力学(aerodynamics)法. 在 Schmidt (1915)等湍流研究工作的基础上, Rossby(1932)较早地 将von Kármán提出的动力相似性和混合长度概念用于 大气和海洋湍流研究. Thornthwaite和Holzman(1942) 进一步明确提出了地表蒸散与风速、温度、湿度等气 象要素廓线存在一定关系, 即空气动力学法. 该方法类 比分子扩散, 认为湍流通量正比于相关要素的梯度, 湍 流扩散系数比分子扩散系数高约 5 个量级. 依托MoninObukhov相似理论(Monin和Obukhov, 1954), 有了梯度 
表 1 现代蒸散测算理论方法的原理、假设或适用条件及优缺点 ${ }^{a)}$

\begin{tabular}{|c|c|c|c|c|c|}
\hline 学科归属 & 测算方法及文献出处 & 基本原理或公式 & 假设或适用条件 & 优势 & 不足 \\
\hline \multirow{4}{*}{$\begin{array}{l}\text { 边界层 } \\
\text { 气象学 }\end{array}$} & $\begin{array}{c}\text { 空气动力学方法 } \\
\text { (Thornthwaite和Holzman, } \\
\text { 1942) }\end{array}$ & $\begin{array}{c}\text { 地表蒸散与风、温、湿等气象要素之间 } \\
\text { 存在明确的垂向廓线关系 }\end{array}$ & $\begin{array}{l}\text { 开阔面, 气象要素 } \\
\text { 垂向梯度分布 }\end{array}$ & 易于实现 & 可移植性差 \\
\hline & $\begin{array}{c}\text { 浴动相关法(Montgomery, } \\
1948 \text { ) }\end{array}$ & $L E=\rho \lambda \overline{w^{\prime} q^{\prime}}$ & $\begin{array}{c}\text { 空气密度脉动忽略 } \\
\text { 不计、平均垂直风 } \\
\text { 速为零 }\end{array}$ & $\begin{array}{c}\text { 理论假设少、 } \\
\text { 原理严谨 }\end{array}$ & $\begin{array}{l}\text { 存在能量不 } \\
\text { 闭合问题 }\end{array}$ \\
\hline & $\begin{array}{c}\text { 通量方差法(Tillman, } \\
\text { 1972) }\end{array}$ & 利用湍流方差和湍流特征, 计算湍流通量 & $\begin{array}{l}\text { 水平均匀平坦地 } \\
\text { 表、不稳定大气 } \\
\text { 条件 }\end{array}$ & $\begin{array}{l}\text { 浴动相关仪的倾 } \\
\text { 斜订正要求较低 }\end{array}$ & $\begin{array}{l}\text { 潜热通量的 } \\
\text { 准确性不如 } \\
\text { 显热通量 }\end{array}$ \\
\hline & 波文比法(Lewis, 1995) & $\beta=\frac{H}{L E}=\gamma \frac{T_{1}-T_{2}}{e_{1}-e_{2}}$ & $\begin{array}{c}\text { 温湿梯度分布明 } \\
\text { 显、垫面均匀、 } \\
\text { 无平流影响 }\end{array}$ & 物理基础明确 & $\begin{array}{c}\text { 使用限制性 } \\
\text { 强 }\end{array}$ \\
\hline \multirow{3}{*}{$\begin{array}{l}\text { 水文 } \\
\text { 气象学 }\end{array}$} & $\begin{array}{l}\text { Penman-Monteith法(Mon- } \\
\text { teith, 1965) }\end{array}$ & $\begin{aligned} L E= & \frac{\Delta}{\Delta+\gamma^{*}}(R n-G) \\
& \rho C_{p}\left(e_{\mathrm{a}}^{*}-e_{\mathrm{a}}\right) / r_{\mathrm{a}}\end{aligned}$ & $\begin{array}{c}\text { 饱和及非饱和下垫 } \\
\text { 面 }\end{array}$ & $\begin{array}{l}\text { 机理性强, } \\
\text { 应用广泛 }\end{array}$ & $\begin{array}{c}\text { 阻抗要参数 } \\
\text { 化, 存在经验 } \\
\text { 性 }\end{array}$ \\
\hline & $\begin{array}{l}\text { Priestley-Taylor法(Priest- } \\
\text { ley和Taylor, 1972) }\end{array}$ & $L E=\alpha \frac{\Delta}{\Delta+\gamma}(R n-G)$ & 地表水分供应充分 & 参数少, 易于应用 & $\begin{array}{l}\text { 经验参数会 } \\
\text { 变化 }\end{array}$ \\
\hline & $\begin{array}{c}\text { 互补相关法(Bouchet, } \\
1963)\end{array}$ & $E T_{\mathrm{a}}+E T_{\mathrm{p}}=2 E T_{\mathrm{w}}$ & $\begin{array}{c}\text { 区域尺度的实际蒸 } \\
\text { 散与潜在蒸发存在 } \\
\text { 互补关系 }\end{array}$ & 无阻抗参数 & $\begin{array}{l}\text { 存在经验 } \\
\text { 参数 }\end{array}$ \\
\hline \multirow{4}{*}{$\begin{array}{l}\text { 陆地 } \\
\text { 水文学 }\end{array}$} & $\begin{array}{c}\text { 零通量面法(Richards, } \\
\text { 1954) }\end{array}$ & 不同时段不同深度的土壤含水量差异 & $\begin{array}{c}\text { 非饱和带零通量面 } \\
\text { 之上水量变化全部 } \\
\text { 为蒸散量 }\end{array}$ & 原理简单 & $\begin{array}{c}\text { 存在侧渗等 } \\
\text { 情况下误差 } \\
\text { 大 }\end{array}$ \\
\hline & $\begin{array}{c}\text { 集水域水量平衡法 } \\
\text { (Brutsaert, 1982) }\end{array}$ & $E=P-Q-\frac{\mathrm{d} w}{\mathrm{~d} t}$ & $\begin{array}{c}\text { 流域尺度水量收支 } \\
\text { 闭合 }\end{array}$ & 原理简单 & $\begin{array}{l}\text { 小尺度上误 } \\
\text { 差大 }\end{array}$ \\
\hline & & $\frac{E T_{\mathrm{a}}}{P}$ & 流域气候民度 年 & & \\
\hline & Budyko法(Budyko, 1948) & $=\sqrt{\frac{E T_{0}}{P} \tanh \left(\frac{P}{E T_{0}}\right)\left[1-\exp \left(-\frac{E T_{0}}{P}\right)\right]}$ & $\begin{array}{c}\text { 净辐射全部转变为 } \\
\text { 潜在蒸发 }\end{array}$ & 简单易行 & $\begin{array}{c}\text { 存在经验参 } \\
\text { 数 }\end{array}$ \\
\hline
\end{tabular}

a) $L E$, 潜热通量; $\rho$, 空气密度; $\lambda$, 水体的汽化潜热; $w^{\prime}$, 垂直风速脉动; $q^{\prime}$, 湿度脉动; $\beta$, 波文比; $H$, 显热通量; $\gamma$, 干湿球常数; $T_{1}-T_{2}$, 两个高 度温度差; $e_{1}-e_{2}$, 两个高度水气压差; $\Delta$, 饱和水气压相对于温度的导数; $\gamma^{*}=\gamma\left(r_{\mathrm{a}}+r_{\mathrm{s}}\right) / r_{\mathrm{a}} ; r_{\mathrm{a}}$, 空气动力学阻抗; $r_{\mathrm{s}}$, 表面阻抗; $R n$, 净辐射; $G$, 土壤热 通量; $C_{p}$, 空气热容量; $e_{\mathrm{a}}^{*}$, 饱和水气压; $e_{\mathrm{a}}$, 空气水气压; $\alpha$, Priestley-Taylor参数; $E T_{\mathrm{a}}$, 实际蒸发量; $E T_{\mathrm{p}}$, 潜在蒸发量; $E T_{\mathrm{w}}$, 湿润环境蒸发量; $E$, 蒸 发量; $P$, 降水量; $Q$, 径流量; $\mathrm{d} w / \mathrm{d} t$, 陆地水储量变化量; $E T_{0}$, 潜在蒸发量; $\tanh$, 双曲正切函数

相似函数 (又称作通量-梯度关系), 空气动力学法也称 为通量梯度(flux gradient)法. 根据Monin-Obukhov相 似性理论, 可以得到近地面层平均气象要素廓线与湍 流通量的泛函关系. 结合风速、温度和湿度的稳定度 修正函数，通过不同高度的平均风速、温度和湿度的 测量，计算摩擦速度、温度特征尺度和湿度特征尺度， 进而计算相应的湍流通量. 这种方法利用气象要素的 垂向梯度观测，易于实现，并成为早期广泛应用的方 法(Dyer, 1974; Lee, 2018). 由于通量-廓线关系存在经
验参数, 不同研究结果存在一定的偏差, 容易导致测算 结果出现偏差.

另外，Tillman(1972)提出利用气象参数的湍流方 差和湍流特征来计算湍流通量, 称为通量-方差相似关 系. 该方法对浴动相关系统的安装与倾斜订正要求较 低，可用于水平均匀、平坦、不稳定条件. 但是，通 量-方差法测算潜热通量的准确性不如显热通量, 其应 用的普遍程度不如浴动相关法(Zhao等, 2010; Ahiman 等, 2018). 
(3) 浴动相关(eddy covariance)法. Montgomery (1948)和Swinbank(1951)等认为, 大气边界层的近地层 湍流交换是地表水热交换的主要机制. 根据湍流统计 理论, 利用风速、温度和湿度等气象要素的脉动数据, 计算相应脉动量的协方差，进而得到地表显热通量和 潜热通量(蒸散量)(Horst和Weil, 1994; Leclerc和Foken, 2014). 20 世纪50年代, 随着声波风速仪的发明, 以及一 系列微气象观测实验和非均匀下垫面湍流观测计划的 实施, 浴动相关测量系统逐渐趋于成熟, 于 20 世纪 90 年 代初在全球开始推广(Foken等, 2012; Baldocchi, 2013). 涡动相关法的理论假设较少, 原理较为严谨完善, 对湍 流交换系数、风廓线形状以及气团浮力作用等都没有 特定假设，是目前平坦均匀下垫面获取地表与大气之 间热量、物质和动量交换应用较多的方法(Aubinet等, 2012), 也是1997年启动的国际通量观测网络(FLUXNET) 常用的观测手段(Baldocchi等，2001). 浴动相关 仪可实施连续观测, 数据持续性和稳定性好, 不影响 植物冠层等周围环境, 但专业维护保养成本较高. 该 方法普遍存在能量不闭合的问题，即潜热通量和显热 通量之和小于地表可利用能量的现象. 一般测算误差 为5 20\%, 在近中性层结条件下相对误差显著增加 (Foken等, 2012), 一般需进行闭合校正.

\subsection{2 水文气象学原理及方法}

水文气象学(hydrometeorology)一词出现相对较 晚, 一般指用气象学方法解决水文学问题, 包括降水和 蒸散等, 属于气象学和水文学的交叉学科, 尚无明确的 学科边界(Bruce和Clark, 1966), 主要研究小时到日尺 度的地气交换现象过程及其统计特征、物理机制和数 学表达(Shuttleworth, 2012). 与边界层气象学不同, 水 文气象学方法注重同时考虑使用大气和下垫面变量, 而不仅仅限于使用大气湍流信息。这类估算地表蒸散 的主要方法包括Penman-Moneith法、互补关系法、

Priestley-Taylor法等.

(1) Penman-Monteith法. 英国气象学家Penman (1948)综合考虑空气动力过程与地表能量平衡原理, 提出利用净辐射、气温、相对湿度、风速等常规气象 观测要素，计算水分充分、供水不受限制条件下的蒸 发公式. 英国科学家Monteith(1965)在Penman公式基 础上, 引入植被气孔阻抗等概念, 提出水分限制条件 下的蒸散计算公式，被称为Penman-Monteith(P-M)方
法，是目前最具机理性的测算方法(Wang和Dickinson, 2012), 均方根误差为 $50 \mathrm{~W} \mathrm{~m}^{-2}$. 由于无法直接测量阻 抗, 需要对其进行参数化, 而现有的参数化方法存在不 同程度上的经验性，因此Penman-Monteith公式仍属于 诊断型模型(diagnostic)(Brutsaert，1982; Shuttleworth, 2007; Liu等, 2012).

(2) 互补关系(complementary relationship)法. 法国 学者Bouchet(1963)最早提出了蒸散互补关系, 即在平 流作用较小的均匀下垫面上, 实际蒸散和潜在蒸散之 间存在着互补关系. 在外界能量输入(净辐射)保持不 变条件下，随着下垫面水分供给减少，实际蒸散减小， 更多的能量成为感热, 温度升高, 从而导致潜在蒸散增 加. 该方法同样不需阻抗参数, 无需植被和土壤参数作 为输入, 可用于资料稀缺区的地表蒸散估算. 然而, 这 种互补关系尚缺乏统一的物理表达式，从起初的对称 型线性关系，先后发展为数种不同的非对称型非线性 关系，在实际应用时存在需要校正的经验参数，均方 根误差约为 $10 \sim 30 \mathrm{~W} \mathrm{~m}^{-2}$ (Han和Tian, 2020). 另外, 不 同的潜在蒸散计算方法也会影响实际蒸散的测算结果 (Brutsaert, 2015; Ma和Szilagyi, 2019).

(3) Priestley-Taylor法. Priestley和Taylor(1972)基 于平衡态蒸散概念, 即地表与大气之间的水气压保持 平衡, 结合地表能量平衡原理, 提出一种相对简明的 蒸散计算公式. 通过引入Priestley-Taylor参数, 避免了 空气动力学阻抗与植被阻抗等复杂的参数化过程及经 验假设等不确定性，仅需要净辐射、土壤热通量和气 温作为输入变量, 易于应用. 虽然Priestley-Taylor公式 非常简单, 且没有考虑气孔阻抗等复杂的机理过程, 但 诸多应用研究表明, 它可用于多种地表条件, 因此成为 使用最为广泛的测算方法之一. Priestley-Taylor参数的 数值随环境条件变化而变化, 在常态情况下处于 1.0 1.5之间,一般取值为1.26(de Bruin, 1983; Wang等, 2004). 在大风和干燥条件下, 该方法有明显的低估; 而 在湿润条件下，高估现象不明显(Cristea等, 2013).

\subsection{3 陆地水文学原理及方法}

陆地水文学发展历史较为悠久, 水量收支平衡是 其最基本的原理. 与气象过程相比，产汇流等水文过 程变化较为缓慢, 多为分钟、小时和日尺度过程. 虽 然蒸散是陆地水循环的一个重要环节，但实际蒸散难 以直接测量, 因此, 常根据其他的水分收支要素, 在田 
间或集水域尺度，利用水量平衡原理，间接计算获得. 田间尺度涉及零通量面法，集水域尺度涉及流域水量 平衡法和Budyko法等(Brutsaert, 2005).

(1) 零通量面(zero flux plane)法. 美国学者Richards(1954)最早提出了零通量面法，即在非饱和土壤 带, 使用位于不同深度的张力计测量土壤水势, 确定水 分通量为零的位置, 由此测算零通量面之上土壤蒸发. 在没有根系吸收、产流和侧渗存在的情况下，通过测 量前后不同时段、不同深度的土壤含水量，利用待测 土柱的水量平衡原理，可以获得该土柱在测量期间的 总蒸发量(Willis，1960；Stammers等，1973). 由于土壤 深层渗漏一般难以定量观测，观测频率一般为日尺度， 该方法大多适用于深层渗漏可忽略的情况，并不适合 作为常规方法广泛使用. 在地下水位接近地表、频繁 强降雨、侧渗未知或不可忽略、深层渗漏较大以及土 壤异质性较大等情况下, 该方法存在较大的误差(Khali1等, 2003).

(2) 集水域水量平衡法. 通过直接测量或间接估算 一定时间内集水域水量平衡的各个分量，包括降水 量、径流量、土壤水分变化量等, 利用水量平衡原理, 求得集水域尺度的蒸散量(Brutsaert，1982)。该方法适 用的空间尺度可以从小斑块 $\left(5 \sim 10 \mathrm{~m}^{2}\right)$ 到大型流域(数 百万平方公里), 适用的时间尺度可以从数周到数年. 该方法的关键在于准确地测量出水量平衡各要素. 在 实际应用中，由于各项估算均存在一定的误差，尤其 是各项都具有空间变异性，而且产汇流形成存在时间 滞后现象，因此在田间尺度等小尺度上，计算得到蒸 散量的误差往往较大.

(3) Budyko法. 苏联气候学家Budyko(1948)认为, 陆面长期平均的蒸散量是由大气对流域的水分供给 (降水)和蒸发能力(潜在蒸散或净辐射)之间的平衡决 定的. 综合水量平衡和能量平衡原理, 获得蒸散量与同 期水量之比与干燥度(潜在蒸发与年降水量之比)之间 的指数关系, 可以用来测算年尺度上的流域蒸散量. 这 一方法简单易行, 尤其是 21 世纪以来, 在大区域气候尺 度研究中获得广泛应用，且可用于分析植被变化对流 域水循环的影响(Zhang等, 2001; Gan等, 2021). 该方 法的主要问题是，采用不同的潜在蒸发公式会在一定 程度上影响测算结果; 另外公式中涉及的经验参数, 其物理意义尚不明晰.

\section{2 观测技术及仪器}

在理论认识发展的同时，仪器技术的发展也推动 了地表蒸散过程的观测，包括蒸发血、蒸渗仪、波文 比仪、浴动相关仪等(表2)。这些仪器具有不同的观测 方式及特点, 在实际运用时需要予以准确把握, 从而正 确地认识观测数据所反映的蒸散变化过程.

(1) 蒸发血. 蒸发血发明于14 16世纪的欧洲文艺 复兴时期, 是蒸散测量历史最为悠久的观测仪器, 测量 水面蒸发, 常用于代表潜在蒸发的大小. 目前在全世界 气象观测场中使用普遍，中国多采用口径 $20 \mathrm{~cm}$ 蒸发血 和E-601B型蒸发皿. 蒸发皿侧壁受辐射和大气热量交 换影响，会造成水分损失. 可采用折算系数将这两种 观测资料衔接使用. 在用于估算湖面蒸发或陆面蒸发 时, 需要结合经验系数进行校正(Ward和Robinson, 2000). 在各类蒸散仪器中, 蒸发血的使用范围最广, 观测数据的持续积累历史最长，常用于分析全球蒸发 变化(Roderick和Farquhar, 2002).

(2) 蒸渗仪. 蒸渗仪通过测量容器内充填土体及植 被的整体重量变化, 利用水量平衡原理, 获得容器在单 位时间内的蒸散量, 是较早测量实际蒸散的重要测量 手段之一，其使用追溯到1688年(de la Hire, 1720; Howell等，1995)，但目前尚未形成蒸渗仪的国际标准 (World Meteorological Organization, 1996). 蒸渗仪的容 器直径通常数米, 深 $1 \sim 2 \mathrm{~m}$ 的填土容器, 埋于地下与地表 持平, 上面可种植植物. 蒸渗仪属于传统的测量仪器, 但造价不菲, 应用受到限制. 为减少测定面积过小所造 成的感热通量差异、底部土壤压力变化带来的土壤水 渗漏量差异等问题, 蒸渗仪建造趋于大型化和精确化.

(3) 闪㐿通量仪. 除了2.1.1节中提到的波文比仪和 浴动相关仪(表2), 近年来推出了闪伢通量仪。苏联学 者Tatarskii(1961)最早提出了波在湍流媒介中传播理 论, 即光闪㷧原理. 闪㐿通量仪是根据近地层大气中不 同湍浴的温度和湿度涨落，引起折射指数脉动和接收 端光强的起伏, 进而推算折射指数及大气的温度湿度 结构参数，并利用Monin-Obukhov相似性理论推算感 热通量和潜热通量. 闪炼通量仪可在较大的空间尺度 (100 1000m)范围测量地-气之间复杂下垫面的感热通 量和显热通量(Wesely, 1976). 自20世纪90年代中期, 闪 炼通量仪迅速发展，并应用于估算存在平流或非均匀 下垫面情况下的大范围区域的地表通量，用于相应网 
表 2 地表蒸散的主要测量手段及其原理、假设或适用条件及优缺点

\begin{tabular}{|c|c|c|c|c|}
\hline 观测仪器及文献出处 & 基本原理或公式 & 假设或适用条件 & 优势 & 不足 \\
\hline $\begin{array}{c}\text { 蒸发㿼 } \\
\text { (Roderick和Farquhar, } \\
\text { 2002) }\end{array}$ & $\begin{array}{c}\text { 根据蒸发血内水位差计算 } \\
\text { 蒸发量 }\end{array}$ & 假设水量变化不受器血质材等影响 & 直接观测 & $\begin{array}{c}\text { 蒸发血侧壁受辐射和大 } \\
\text { 气热量交换影响 }\end{array}$ \\
\hline $\begin{array}{c}\text { 蒸渗仪 } \\
\text { (Howell等, 1995) }\end{array}$ & $\begin{array}{c}\text { 利用水量平衡原理, 测量容器 } \\
\text { 内充填土体及植被的整体重量 } \\
\text { 变化 }\end{array}$ & $\begin{array}{l}\text { 自重变化不受蒸散以外因素影响, } \\
\text { 适用于土壤或覆有植被的地表蒸散 }\end{array}$ & 直接观测 & $\begin{array}{l}\text { 制作成本高, 观测空间尺 } \\
\text { 度小, 边界效应强 }\end{array}$ \\
\hline $\begin{array}{c}\text { 波文比仪 } \\
\text { (Todd等, 2000) }\end{array}$ & $\begin{array}{c}\text { 测量空气温度和湿度的梯度 } \\
\text { 变化来计算地表蒸散量 }\end{array}$ & $\begin{array}{c}\text { 能量闭合、热和水气的传输系数相等, } \\
\text { 下垫面相对均匀且无平流影响 }\end{array}$ & $\begin{array}{l}\text { 方法简单且 } \\
\text { 精度较高 }\end{array}$ & $\begin{array}{l}\text { 气温梯度和湿度梯度接 } \\
\text { 近于零的情况下误差大 }\end{array}$ \\
\hline $\begin{array}{c}\text { 涡动相关仪 } \\
\text { (Horst和Weil, 1994) }\end{array}$ & $\begin{array}{c}\text { 测量空气湍流的脉动变 } \\
\text { 化来获得蒸散量 }\end{array}$ & $\begin{array}{c}\text { 空气密度脉动忽略不计、平均垂直 } \\
\text { 风速为零, 平坦均一下垫面 }\end{array}$ & $\begin{array}{l}\text { 原理完善, 长期定 } \\
\text { 位连续观测 }\end{array}$ & 专业维护保养成本高 \\
\hline $\begin{array}{c}\text { 闪炼通量仪 } \\
\text { (Moene等, 2009) }\end{array}$ & $\begin{array}{c}\text { 根据大气中光闪胨来推算 } \\
\text { 大气中的热通量 }\end{array}$ & $\begin{array}{c}\text { 湍流相似性, 单一均匀、非均匀 } \\
\text { 以及复杂地表 }\end{array}$ & 观测足迹相对稳定 & $\begin{array}{c}\text { 用于较大空间尺度、存 } \\
\text { 在平流、非均匀下垫面, } \\
\text { 涉及参数多 }\end{array}$ \\
\hline $\begin{array}{c}\text { 茎流计 } \\
\text { (Granier, 1987) }\end{array}$ & $\begin{array}{c}\text { 加热植物茎干, 测量茎流速率, } \\
\text { 进而计算单株植被蒸腾量 }\end{array}$ & 可用于短时间观测 & $\begin{array}{l}\text { 无需标定, 直接测 } \\
\text { 定, 可靠性高 }\end{array}$ & 可靠性高, 对植物有害 \\
\hline $\begin{array}{c}\text { 原位同位素技术 } \\
\text { (Yakir和Sternberg, } \\
\text { 2000) }\end{array}$ & $\begin{array}{c}\text { 根据氢氧同位素的分馏现象, } \\
\text { 定量区分蒸散组分 }\end{array}$ & 下垫面均一 & 区分蒸散组分 & 下垫面均匀, 耗材较大 \\
\hline
\end{tabular}

格尺度的遥感产品及水文大气模式的结果检验 (Moene等, 2009; Zeweldi等, 2010; Liu和Xu, 2019). 仪 器观测质量取决于天气状况、空气折射指数、信号强 度以及后处理过程. 根据所采用的孔径大小不同, 闪伢 通量仪可分为小孔径闪伢仪、大孔径闪伢仪和超大孔 径闪炼仪(Foken, 2008). 早期发展的光学波段闪炼仪, 特别是大孔径闪炼仪, 只能获取感热通量. 近十多年发 展的微波(毫米波)闪炼仪，与光学闪秌仪结合(所谓双 波长系统Optical-Microwave Scintillometer, OMS), 可 同时获取感热通量和潜热通量，是测算田块到景观尺 度地表蒸散的有效方法.

(4) 茎流计. 茎流计是通过加热植物茎干来测量茎 流速率进而计算单株植被蒸腾量的一种仪器. 根据测 定原理不同，可分为热脉冲法、热平衡法、热扩散法 和激光热脉冲法. 德国植物生理学家Huber(1932)最早 提出利用热脉冲法测定单株植被的茎流速率, Marshall (1958) 从仪器结构和测量原理方面做了全面的改进. 后经不断地完善，已广泛用于研究不同地区、不同类 型单株树干的茎流特征(Swanson和Whitfield，1981; Granier, 1987; Dye等, 1996, Clausnitzer等, 2011).

Lewis和Cornish(1933)发现氢氧同位素在水的不 同相态存在分馏现象. 根据同位素质量守恒原理, 可以 利用氢氧稳定同位素技术定量区分地表蒸散组分(土 壤蒸发和植被蒸腾等)(Keeling，1958，Craig和Gordon,
1965; Walker和Richardson, 1991; Yakir和Sternberg, 2000). 20 世纪90年代以来，水气同位素原位连续观测 成为可能, 广泛用于区分蒸散水气的不同来源, 以及蒸 散组分比例; 结合浴动相关仪的高频观测技术, 可实现 较为精确的蒸散组分量化区分(Yakir和Wang, 1996; Phillips和Gregg, 2001; Beyer等, 2020).

\section{0 世纪90年代以来蒸散研究的发展态势 及联系特征}

20 世纪 90 年代以来，迅速发展的浴动相关系统和 卫星遥感等观测手段, 跨越寒带-热带、干旱-湿润等 气候区, 涵盖了不同下垫面类型, 极大地拓展了蒸散观 测的时空维度(Baldocchi等, 2001; 刘元波等, 2016), 借 助于互联网的发展(Brügger和Milligan，2019), 从现象 认识和理论方法等方面推动了蒸散研究. 下面围绕地 表过程观测、自然现象发现、理论方法探索三个方面 予以简述，而不涉及陆面过程或水文模型等广泛应用.

\section{1 地表蒝散观测急剧拓展成网, 卫星遥感实现全 球无缝衔接}

蒸发血测量所在环境条件下的水面蒸发, 往往代 表的是蒸发潜力. 蒸渗仪测量容器内土柱或植株的实 际蒸散，但很难普及不同类型下垫面. 直到 20 世纪 80 
年代，随着红外光谱仪电子计算机和大容量数据存储 技术的推广应用，浴动相关系统才得以快速发展成熟， 应用于不同下垫面蒸散观测，并逐渐成为地表蒸散长 期连续监测的观测仪器, 涵盖了水体、湿地、森林、 农田、草地、裸地、城市等各种地被类型(Baldocchi 等, 2001). 随着BOREAS(the Boreal Ecosystem-Atmosphere Study)等陆地观测国际大型科学计划的实施与 推进(Sellers等, 1997), 区域性地表通量观测站网AmeriFLUX和EuroFLUX联合发展为全球性通量观测网站 (FLUXNET). 作为地球观测卫星计划(the Earth Observation Satellite, EOS)的遥感产品检验的一部分, FLUXNET获得了美国国家航空航天局(National Aeronautics and Space Administration, NASA)的资助(Baldocchi等，2001). 目前FLUXNET还包括FLUXNETCanada、CarboEurope、ChinaFLUX、AsiaFLUX、 KoFLUX、OzFLUX等区域站网(fluxnet.ornl.gov)。注 册观测站点多达 900 余处，在测站点 560 余处，较为全 面地分布在全球各种气候区和各种生物群落类型中, 覆盖范围从 $70^{\circ} \mathrm{N}$ 至 $30^{\circ} \mathrm{S}$, 包括不同气候和水分梯度下 的森林、农田、草地、荒漠和水体等不同地表类型. FLUXNET提供每个站点包括地表覆被状况在内的自 然地理要素信息. 这些站点普遍采用微气象站、浴动 相关系统及相关仪器设备, 观测地表微气象要素, 地 气间水气、能量和二氧化碳交换通量以及地表覆被 变化等, 并进行数据共享. 最早的通量观测数据见于 1985年, 其中可共享数据的基本采样频率为半小时平 均值, 积累了 30 年的地表要素及水热通量观测数据. 近二三十年来，在国家自然科学基金委员会、科技部 等相关科研项目支持下，中国数十家机构或研究团队 采用涡动相关系统(EC), 在全国尺度上设置了特定区 域的长期观测样带，包括不同区域的森林、草原、湖 泊观测站网，其中ChinaFLUX是中国最大的通量观测 网络(于贵瑞等, 2014). 除站点观测之外, 还建立由数 台或数十台浴动相关系统组成的通量观测矩阵，以探 究空间异质性等对地表水热通量的影响(Engelmann 和Bernhofer, 2016; Liu等, 2018). 这些观测不但获取 了大量的不同地表的蒸散特征资料，同时也为模型对 比、新理论建模及精度检验(直接检验、间接检验、 交叉检验)奠定了坚实的现实基础(刘元波等, 2016; 张 圆等, 2020).

在卫星遥感对地观测方面，美国1978年发射的热
容量制图卫星HCMM(Heat Capacity Mapping Mission)和极轨气象卫星泰洛斯TIROS-N(Television Infrared Observation Satellite)提供的红外数据促发了地表 蒸散遥感反演研究(Price, 1980; Jackson, 1985). 近四十 年来，随着经典蒸散模型和航天航空遥感技术的日趋 成熟，蒸散遥感方法逐步发展起来. 现有的蒸散遥感 方法，基本上都是通过建立某一(些)谱段辐亮度或复 合指数与蒸散测量值之间的统计关系，或者基于某个 蒸散模型所表达的测算关系, 来实现遥感反演(刘元波 等, 2016). 按照所依据的蒸散测算模型(正演模型), 主 要包括基于Priestley-Taylor(P-T)模型、基于P-M模 型、基于互补关系法、基于能量剩余法的反演方法 等, 主要为大气边界层物理模型. 基于相同或不同的地 表蒸散模型, 目前已经发展了数十种蒸散反演方法, 并 已生产发布了多款全球地表蒸散产品(Wang和Dickinson, 2012; 张圆等, 2020). 陆表能量平衡算法SEBAL (Surface Energy Balance Algorithm for Land)(Bastiaanssen等, 1998)、植被指数-地面温度三角关系法(Jiang和 Islam, 1999)、地表能量平衡系统法SEBS(Surface Energy Balance System)(Su, 2002)等, 都是应用广泛的反 演方法. 按照所使用数据的传感器类型, 蒸散遥感反演 方法分为可见光-近红外、热红外、被动微波及组合 方式, 其中大多数方法采用了可见光-近红外和热红外 波段数据(Glenn等, 2010; 刘元波等, 2016).

总之, 站点高频观测手段和航天航空遥感技术结 合，构筑了从植物气孔到叶片、植株、冠层、景观、 流域等多尺度测算手段，推动着地表蒸散的过程观测 走向横向综合; 同时，也对地表蒸散的机理解析走向 纵向深化等, 提供了基础背景.

\section{2 新的观测事实促进机理解析, 研究重点转向非 均匀下垫面}

随着地表蒸散观测在时空维度上不断延展，一方 面, 在单一下垫面获得了新的观测事实, 例如, 地表夜 间蒸散和蒸散迟滞现象等(Schmugge和André，1991; Daley和Phillips, 2006); 另一方面, 研究重点从早期的 单一、均匀、平坦下垫面, 转向非均匀下垫面, 包括不 同下垫面之间的相互作用(绿洲冷岛效应)、下垫面自 身变化的影响(水陆转捩效应)等(Zhao和Liu, 2017). 这 些观测发现，对经典相似性理论和地表蒸散理论等提 出了新的挑战, 简述如下. 


\subsection{1 地表夜间蒸散}

在水文气象、生物气象、生态水文、陆面过程、 植物生理等经典论著中, 通常认为陆面夜间蒸散量(或 凝结量)处于极低水平，可忽略不计(Brutsaert，2005; Zeppel等，2014)。借助于植物茎干液流计、气体交换 仪、浴动相关系统、蒸渗仪、同位素仪等观测技术, 21 世纪以来陆续发现, 除了土壤蒸发, 不同生境的植物 普遍存在着夜间蒸腾现象(Daley和Phillips, 2006; Caird 等，2007; Moore等，2008; Ogle等，2012; Cirelli等， 2016). 植物夜间蒸腾量约占全天总量的10 25\%(Caird 等，2007; Zeppel等，2014), 在干旱环境下会超过30\% (Ogle等, 2012; Cirelli等, 2016). 在干旱环境下, 白天植 物叶片气孔未完全开启而保持较低蒸腾速率, 夜间气 孔未完全关闭而存在一定程度的叶片蒸腾. 夜间蒸腾 量大小取决于气孔导度、叶-气间的饱和水气压差、 冠层结构和大气混合状况等因素，调控植物冠层与大 气之间的水分交换，起着保持和恢复植物体内水分平 衡、运输和分配营养物质等重要作用. 由于夜间湍流 不发育, 难以满足湍流相似性理论假设, 对空气动力 学法等相关测算方法而言, 存在较大的精度问题. 虽 然夜间蒸散量水平较低，但凸显了经典理论方法的局 限性，也是浴动相关系统等蒸散观测数据分析中面临 的突出问题.

夜间空气冷却到露点或大气水气饱和时, 会出现 凝结现象，凝结量可占年降水量的 5 23\%，一般干旱 区的占比要高于湿润区，同一地区干旱年份的占比要 高于湿润年份(Jacobs等, 2006; Uclés等, 2014; Zhuang 和Zhao, 2017), 是许多陆地生态系统的重要水源, 对动 物、植物、微生物而言, 具有重要的生理生态作用 (Burgess和Dawson, 2004; Rao等, 2009; Eller等, 2013; Kidron等, 2014).

\subsection{2 蒸散相位差现象}

地表净辐射或可利用能量转化为潜热和显热等热 量, 并非瞬间完成, 在时序变化上往往表现出非同步特 征, 称为地表能量与蒸散之间的相位差异现象. 这种现 象直到 20 世纪 90 年代初，才逐渐引起人们的关注 (Schmugge和André, 1991; Crago, 1996; Liu和Hiyama, 2007). 蒸散量的相位变化可先于或迟于风速、温度和 湿度等环境变量, 在相空间中分别表现为顺时针或逆
时针的迟滞环．其旋转方向、形状、面积等属性，随 着所在地点和下垫面而异，包括草地、绿洲、森林等， 在湖泊上表现得尤其突出, 潜热通量可滞后于净辐射 量4 5个小时(Wang等, 2014; Zhao和Liu, 2017; Yu等, 2019; Cui等, 2020). 湖泊具有粗楉度小、反照率低、 比热容大、热传导率低等物理属性, 加上流体特有的 湍流扩散特性，使其具有很大的储热潜力，导致湖泊 蒸发与净辐射在日尺度上出现明显的相位延迟, 在季 节尺度上可达数月, 且湖泊越深, 相位延迟越大(Gianniou和Antonopoulos, 2007; Blanken等, 2011). 中国的 青海湖、色林错、太湖和鄱阳湖都有相关报道(Wang 等, 2014; Li等, 2016; Zhao和Liu, 2017; Guo等, 2019). 不考虑相位差异, 将会导致蒸散测算的高估或低估, 均 方根误差可达 $100 \mathrm{~W} \mathrm{~m}^{-2}$. 包括P-M公式在内的几乎所 有的蒸散测算方法都未明确地刻画迟滞效应，在公式 计算结果与观测数值之间存在的相位差异, 成为蒸散 测算难以避免的不确定性来源之一(Cui等, 2020).

\subsection{3 非均匀下垫面岛屿效应}

在非均匀下垫面上，不同冷热下垫面之间可产生 动量、能量和水分交换, 称为平流(Philip, 1959; McNaughton, 1976). 在蒸散测算公式中一般并未明确 包含此项, 可作为边界条件或能量附加项引入(Itier等, 1994)。平流现象在干湿交界处表现突出，包括荒漠绿 洲边缘、水库周边等. 早期研究多集中于干旱区的灌 溉地周边，来自干区的热平流显著地提高干湿交界处 的蒸散, 故又称为局地平流(local advection)或前缘效 应(leading edge effect)(Spronken-Smith等, 2000).

在绿洲或灌区尺度上，通过湍流交换、平流过程 或局地环流与周围环境进行物质和能量交换, 绿洲内 部形成的逆温稳定层结，会抑制湍流的发展，并抑制 植物蒸腾和地表蒸发, 从而维持绿洲气候的稳定, 被 称为绿洲冷岛效应. 早期的绿洲效应(oasis effect)一词 是指局地前缘效应(de Vries, 1959), 直到20世纪80年代 末才实现绿洲尺度上的观测模拟(高由禧，1989; Taha 等, 1991). 与此类似, Manley(1958)最早提出了城市热 岛概念，指城市下垫面性质改变、大气污染及人工废 热等引起热量集聚，城区温度明显高于周边郊区而形 成高温 “岛屿”. 无论是绿洲冷岛效应还是城市热岛效 应，本质上都是由下垫面物理性质的不均匀性而导致 的热力和动力非均匀性, 引起平流甚至二次环流, 进 
而引起地表蒸散的增强或减弱变化，用P-M公式等传 统测算方法很难处理，需要更密集的观测站点和时空 分辨率更高的数学模型. 另外, 城市具有高度的三维 空间异质性，如何运用传统方法进行精准测算三维立 体的蒸散量，是当前面临的一个挑战(Arnfield，2003; Qiu等, 2020).

\subsection{4 下垫面变化引起的转捩效应}

下垫面除了具有空间异质性外，在时间上也会发 生日或季节变化, 例如, 植被季相变化, 北方夏季的草 地变成冬季的裸地，这会导致植被反照率和冠层热储 存效率等性质发生改变, 进而影响地表蒸散变化过程. Zhao和Liu(2017)利用浴动相关系统，在具有水陆季相 转换特征的鄱阳湖区，观测到两种不同类型的水热通 量日变化模式，一种是丰水期的水相模式，潜热通量 和显热通量的日变化周期微弱; 另一种是枯水期的陆 相模式，潜热通量和显热通量的日周期变化明显。处 于水相模式的蒸散，主控因素是风速和净辐射; 而处 于陆相模式的蒸散, 主控因素是净辐射和温度(Zhao和 Liu，2018). 水陆转捩过程的初步分析表明，随着湖泊 下垫面变化, 潜热通量与环境要素之间的迟滞效应, 在 迟滞环的旋转方向、形状和面积上，也都发生了相应 的变化(Cui等，2020). 下垫面转捩效应本质上仍然是 下垫面物理属性改变而导致的地表热力和动力过程变 化, 可结合陆面过程模型等能反映时空变化过程的数 值模拟手段进行分析．在发展新的蒸散测算方法或进 行地表真实性检验时，不但要考虑下垫面的空间非均 匀性，也要考虑下垫面物理属性的时间变化，包括伴 随迟滞效应变化所引起的计算结果与观测数值之间的 相位差异.

\section{3 拓展经典理论方法的适用条件, 探索新的蒸散 测算理论方法}

经典的蒸散理论方法要求下垫面平坦均匀. 在非 均匀下垫面应用时，例如卫星遥感数据或陆面过程模 型，面临着适用性问题。需要借鉴、发展或利用已有 方法，加以合理拓展或校正，为此发展了物理型方法 (如土壤-植被双源模型)和统计型方法(如湍流间歇订 正法). 随着新的观测事实逐渐被发现，经典理论方法 的局限性日益凸显, 以及新的学科理念的不断发展, 促 使人们探求更为完备的物理表达式或新的主导方程,
从而催生新的理论萌芽，这方面的探索包括表面更新 模型(Paw U等, 1992)、地表三温模型(Qiu, 1996)、最 大熵增蒸散模型(Wang等, 2004)、非参数化蒸散模型 (Liu等, 2012)等.

\subsection{1 土壤-植被双源模型(two-source model)}

随着大尺度陆面过程模式和卫星遥感反演精度要 求的不断提高, 下垫面非均匀性的影响成为不可回避 的问题. Shuttleworth和Wallace(1985)最早明确地提出, 将非均匀下垫面分为土壤和植被两大类，采用经典的 单源P-M模型，分别考虑水分与湍流通量的传输过程, 进行耦合求解．Norman等(1995)和Anderson等(1997) 则进一步发展了这一方案，根据连接方式的不同，将 双源模型分为并联和串联两类进行处理. 在稀疏植被 覆盖下, 植物叶片下层土壤裸露, 双源模型比单源模 型更符合实际情况，获得了广泛的应用，包括大陆乃 至全球尺度(Zhang等，2010). 双源模型虽然可以实现 土壤蒸发与植被蒸腾之间的组分分离, 但涉及阻抗等 参数较多, 增大模型测算的不确定性, 影响该方法的 测算精度，难以普及或取代单源模型( $\mathrm{Li}$ 等，2009; Wang和Dickinson, 2012). 另外, 下垫面不但包括土壤 和植被, 还包括水域、冰雪面、岩面、建筑用地等, 也 需要予以考虑.

\subsection{2 湍流间歇作用订正法}

20 世纪 90 年代, 大气湍流研究由早期的平坦、均 一下垫面的探索，向复杂下垫面条件拓展，湍流间歇 作用及影响更为突出，特别是稳定边界层湍流间歇形 成机制成为前沿科学问题(Sun等，2004). 湍流间歇与 复杂下垫面上的大气流场结构，地形激发的重力波、 孤波和类波事件以及其他各种复杂运动形式等有关 (Mahrt, 2007，2010)，湍流间歇性可以描述为: 在相对 较弱的脉动甚至层流的时段出现短暂的湍流运动 (Mahrt，1999)。强稳定层结条件下的湍流间歇过程不 能用Richardson数描述，湍流特征量与Richardson数失 去联系(Mahrt，2010), 湍流统计特征的相似性关系与 观测存在很大偏离(Grachev等, 2015), 影响模式参数 化方案计算湍流交换. Baldocchi等(2001)明确指出稳 定层结湍流通量的观测与分析，是影响通量观测计划 (FLUXNET)整体观测结果的最大制约因素. 可见，因 湍流间歇现象的存在，引起Monin-Obukhov相似性理 
论应用条件失效，传统意义上获取的湍流运动信号包 含了纯湍流运动，也包含了非湍流成分，导致湍流特 征和湍流输送分析出现偏差, 利用气象要素原始脉动 计算得到的湍流参量存在高估的可能，在解析蒸散过 程和测算蒸散量时，应考虑湍流间歇的作用及对湍流 通量的影响，剔除非湍流运动影响，以确定“真”湍流 通量(Wei等, 2018; Ren等, 2019).

\subsection{3 表面更新(surface renewal)法}

在化工领域, Higbie(1935)最早提出气-液两相分子 都有可能被湍流携带而界面不断地被更新. 这一传质 理论直到20世纪90年代才被Paw U等(1992)用于气象 学领域. 表面更新法认为, 湍流运动导致温度和湿度等 标量浓度产生斜坡形式的升降变化，可用结构函数等 表示. 利用温度湍流的高频数据, 通过求算显热通量, 再利用地表能量平衡方程, 进而获得潜热通量. 表面更 新法在湍流过程描述上既不同于浴动相关法，也不同 于湍流间歇理论，在物理过程上三者是否存在关联尚 不得而知，目前应用也不普遍. 现有研究表明，表面更 新法在蒸散测算上精度非常接近浴动相关法，既可用 于稳定条件也可用于非稳定条件，但在高湿条件以及 高风速条件下存在较大的问题(Zhao等, 2010; Hu等, 2018).

\subsection{4 地表三温(three-temperature)模型}

Qiu等(1998)通过引入参考土壤(水分蒸发量为零 的土壤)和参考植被(水分蒸腾量为零的植被), 采用类 似于经典P-M模型的推导思路, 既避免了地表阻抗参 数，又获得了一个简洁的解析型表达式，基于观测站 点的检验均方根误差为 $0.2 \mathrm{~mm} \mathrm{day}^{-1}$. 该模型仅需净辐 射、土壤热通量、三种温度(气温、表面温度和参考 表面温度)作为输入数据, 即可分别计算出土壤蒸发量 和植被蒸腾量, 称为地表三温模型. 由于三温模型不需 要输入空气动力学阻抗和冠层特征(如冠层高度)等参 数, 避免了因气象参数(如风速)插值和地表分类错误 等引起的不确定性. 在空间上，已被用于干旱半干旱 区的自然下垫面、亚热带城市草坪和灌从蒸散及其组 分的遥感反演(Yan和Qiu, 2016; Qiu等, 2017; Xiong等, 2019; Zou等, 2019). 这一模型的重要意义在于, 即使采 用经典P-M模型的相似推导思路，也能避开难以测量 的阻抗参数. 模型的应用难点在于, 如果实际观测(或
遥感影像)中不存在蒸散为零的参考土壤或植被, 其测 算精度存在较大的不确定性.

\subsection{5 最大熵增(maximum entropy production)模型}

出于对地表阻抗参数的物理意义的疑虑, Wang等 (2004，2007)借鉴贝叶斯概率论、信息熵概念、非平 衡态热力学和湍流相似性等理论基础, 提出了最大熵 增地表蒸散模型, 即以地面净辐射、地表土壤水含 量、地表温度及感热通量, 分别表达能量供给、水分 供给、蒸发潜力和湍流水气输运等四个影响蒸散过程 的物理机制，在任意水文气象条件下的地-气相互作用 使得蒸散速率达到最大, 从而得到这个四要素函数在 能量守衡约束下的极值解. 与实际观测数据进行对比 检验表明, 该模型测算误差为 40 60 $\mathrm{W} \mathrm{m}^{-2}$ (Wang和 Bras, 2011). 它具有解析型表达式, 简单易用, 地表辐 射、表面温度、表面比湿等模型输入变量易于实地观 测获取, 既可以用于气象站实地业务测算, 也可用于遥 感反演. 这一蒸散模型已被用于遥感反演大尺度地表 水热过程, 并已尝试用于改进气候模式的参数化方案 (Chen等, 2017; Wang等, 2019).

\subsection{6 非参数化(non-parametric)模型}

同样出于对阻抗参数物理意义的疑虑, Liu等 (2012)另辟蹊径, 采用广义哈密尔顿原理, 把地面温度 视为广义哈密尔顿量, 结合地表能量平衡原理和平衡 态蒸发概念, 推导出测算地表潜热和显热通量的解析 型表达式. 模型输入参数包括净辐射、土壤热通量、 气温和地表温度, 所有输入变量均可实地测量, 不涉 及经验性参数, 故称为非参数化蒸散测算模型. 运用 国际通量网站(FLUXNET)观测数据，除去林地观测站 难以确定地面温度，其他站包括冻原、荒漠、草地、 农田等类型, 模型测算误差约为 $(10.3 \pm 20.2) \mathrm{W} \mathrm{m}^{-2}$ (Liu 等, 2012). 不但可用于实地测算, 也可以用于遥感反演. 黑河流域的观测检验和遥感反演应用表明，均方根误 差为 $20 \sim 50 \mathrm{~W} \mathrm{~m}^{-2}$, 在湿润下垫面会出现一定程度的低 估，在干旱下垫面则会出现高估(王宁等，2016); 在时 序变化上, 反演结果的稳健性突出(Pan等, 2017, 2020).

\section{4 蒸散测算理论方法的发展展望}

陆域蒸散研究历史悠久, 蒸散下垫面多样性及宏 
观复杂性, 使蒸散测算存在很大不确定性, 成为无数地 表过程定量研究的共同制约瓶颈. 随着对地观测全球 化和地学研究日趋定量化, 这一瓶颈效应日益凸显. 如今面临着全球变温和人类活动所引起的一系列人类 生存环境问题, 比以往任何时候都更需要重新认识蒸 散这一古老的命题. 21 世纪以来, 新的研究理念、技 术方法、观测事实不断涌现, 新的理论维形蓄势待发. 目前蒸散测算不确定性主要来源于最基本的物理过程 认识不足, 蒸散广延性延伸带来的时空异质性以及现 有观测系统的局限性, 这三个方面也是未来研究的主 要突破方向.

\section{1 吸纳新现象, 发展新理论}

如前所述, 近三十年来捕捉到的新的观测事实, 包 括夜间地表蒸散、蒸散迟滞现象、非均匀下垫面的湍 流间歇、平流关联的岛屿效应、下垫面转捩效应等, 用经典的湍流理论或主流的地表蒸散理论等难以解 释, 局限性日益凸显, 呼唤新的理念思路或认知角度. 以大气边界层湍流为例, 在稳定层结条件下, 不能沿用 传统的方式来描述强稳定边界层, 现有的湍流通量计 算结果与实际不完全符合等(Wei等, 2018). 以夜间蒸 散为例, 无论是观测技术还是物理模型, 要确定夜间植 物蒸腾量, 面临着不少挑战, 尤其是如何从植物茎干液 流中区分植物体组织补水与叶片蒸腾失水、不同观测 手段的不确定性等, 都是限制瓶颈(Ogle等, 2012; Cirelli等, 2016).

从应用角度来看，除了农业灌溉等各类生产实践， 人们在各类模型对比分析研究的基础上, 运用经典蒸 散模型发展陆面过程模式. 从始于 20 世纪 60 年代的第 一代水箱模式, 经历了第二代土壤-植被-大气传输模 式、第三代考虑碳循环的生物化学模式, 陆面过程模 式已经有了近六十年的发展(Pitman, 2003). 然而, Haughton等(2016)采用相同的FLUXNET通量观测数 据和精度评估指标, 对比分析了八种陆面过程模式, 最 终指出: 当前陆面过程模式的主要误差仍然源于如何 将地表可利用能量分为显热和潜热(蒸散), 而非其他 因素, 这正是蒸散理论方法的核心内容所在. 显而易 见, 除了新的观测事实, 陆面过程模式的检验研究表 明, 经典蒸散模型的根本问题在如何将可利用能量准 确分配为显热和潜热通量, 需要进一步提升完善 (Haughton等, 2016).
回溯历史, 经典的蒸散测算模型的主要理论依据 包括质量、能量、动量守恒等基本物理定律, 以及流 体力学和平衡态热力学的基本理论. 随着物理学从牛 顿经典力学范式, 走向爱因斯坦相对论范式, 再到当今 风靡世界的量子力学范式, 作为跨越多学科的蒸散研 究, 更需要汲取当代科学的新理念, 借鉴混沌力学、非 平衡态热力学等处理非线性及复杂性问题的学科知识, 不断地拓展新思路, 推进蒸散测算理论方法向纵深发 展. 表面更新法(Paw U等, 1992)、地表三温模型(Qiu, 1996)、最大熵增模型(Wang等，2004)、非参数化模型 (Liu等, 2012)等, 虽然所依据的原理和输入变量及主导 方程不尽相同, 但都全力规避使用经典理论方法中的 阻抗等经验参数, 都是值得肯定的艰难探索与积极尝 试. 这些新的理论方法, 从各自不同的角度表明, 经典 的地表蒸散模型有待发展完善, 甚至重建.

总之, 发展新的蒸散理论方法, 需要全面吸纳新的 观测事实, 从最基础的物理原理出发, 发展更能反映蒸 散物理本质的共性方程, 甚至统合各类不同的方法, 从 而实现根本性突破. 在大数据时代, 将相关的物理约束 条件引入统计型机器学习法, 有助于更好地寻求蒸散 形成的物理要素(Zhao等, 2019). 当然也要看到, 提出 全新的理论方法, 往往并非一帆风顺, 需要历经一个从 初步认可、大量检验、反复实践, 到全面接受的长期 过程.

\section{2 关注非均匀下垫面多尺度的蒸散理论和方法}

现有的蒸散测算理论方法及技术手段, 大多以均 匀下垫面为前提假设条件. 在现实条件下, 从极地到 赤道, 从沙漠到湖泊, 下垫面等自然状况时空差异大, 而蒸散几乎无所不在, 构成蒸散测算的尺度不确定性.

下垫面非均匀性所导致的蒸散变化过程是深入认 识其形成机制和开展尺度效应研究的重要前提. 经过 长足的发展, 在观测方式上, 从植物气孔到叶片、植 株、冠层、景观、流域乃至全球, 运用各式点位高频 观测仪器、无人机遥感、卫星遥感等新技术, 已经具 备了多尺度、多方位、连续的蒸散监测能力. 以湍流 观测为例, 利用浴动相关系统, 结合足迹(footprint)模 型, 可以建立足迹范围内的通量分布变化过程; 结合 同步观测的大孔径闪㷧仪, 可评估数十米至数千米级 的空间异质性作用(Liu等, 2011), 包括绿洲冷岛和城 市热岛等岛屿效应, 同样也可以拓展到下垫面转捩效 
应. 以茎流计为例, 结合树木胸径、叶面积、边材面积 等生物学参数或茎流在胸径径阶的分布特征, 实现从 单株植物向群体林分水平的多尺度蒸腾量测算(Cermak和Nadezhdina, 1998; Chabot等, 2005). 虽然多尺度 的观测能力已经具备, 但要利用不同仪器实现可靠的 尺度效应观测, 也面临着一系列的问题. 例如, 在同一 类仪器不同型号之间、不同类型仪器之间, 各自所观 测变量之间存在多大的差异, 这些差异在多大程度上 影响尺度效应的观测可靠性, 等等, 是基于观测系统 开展尺度效应研究所面临的基本前提.

擞开仪器观测相关的误差问题，利用不同尺度间 的转换规律, 建立尺度转换公式或函数, 是将均匀下 垫面理论方法拓展到非均匀下垫面的一个有效途径. 尺度转换在理论上可补偿地表空间异质性所带来的不 确定性. Shuttleworth和Wallace(1985)提出的土壤-植被 双源模型，有效地拓展了经典物理模型所采用的单一 下垫面假设，更好地处理了非均匀下垫面情况. 这种 基于物理原理的尺度转换方法，亦可以推广到两个以 上的多种下垫面情况，有待借助于多尺度观测来予以 实现. 在转换方式上，有尺度上推和尺度下推 $(\mathrm{Wu}$ 等, 2000). 不论哪种方式，尺度转换既可以是基于物理原 理的, 也可以是基于统计的. 基于统计的尺度转换, 主 要依托不同尺度上物理变量之间的统计关系( Liu等, 2016). 基于物理原理的尺度转换, 需要建立在严格的 物理原理之上，既包括能量守恒、质量守恒和动量守 恒等尺度不变律，也包括描述该自然过程的主导方程 (Liu等, 2006), 例如反映蒸散过程的经典模型. 若主导 方程存在经验参数, 即使采用严格的数学物理推导, 如 果不能消除经验参数, 也会影响建立尺度转换关系的 可靠性及普适性. 因此, 发展具有严格物理基础的尺 度转换公式或函数, 首先需要消除主导方程中的经验 参数. 由于蒸散过程的复杂性以及经典模型的不确定 性, 目前蒸散尺度转换方法多局限在统计型或统计-物 理混合型模型(Nouri等, 2020). 深入研究物理型尺度转 换是从理论上解决非均匀下垫面问题的必然趋势.

\section{3 研发蒸散观测新技术, 促进多学科交叉联合试验}

从蒸散理论方法的历史发展过程看, 无论在过程 认知还是理论推演方面, 都离不开同期的观测试验水 平, 观测能力扮演了重要的时代角色. 从学科角度来 看, 边界层气象学、水文气象学、陆地水文学的基本
观测手段各有特点，基本观测时间不尽相同，分别为 秒、时、日. 这种观测差异是造成三个学科在蒸散理 论发展上各有侧重的重要因素之一, 不利于理论融合. Cuxart和Boone(2020)提出, 要研发具有高频观测能力 的蒸渗仪, 一方面可以与浴动相关发展系统进行观测 结果比较, 另一方面可以弥补浴动相关系统在夜间和 雨雾天观测的不足, 为大气边界层研究发展一个新的 途径. 此外, 高频蒸渗仪还将有助于回答湍流相似性 理论存在的一些问题, 如能量不闭合问题. 除了蒸渗 仪, 也可以基于近些年提出的新的测算原理方法, 研 发新的观测系统. 例如, Qiu等(2020)使用地表三温模 型法, 采用热红外成像仪, 估算了单株植被的三维空 间蒸散发量. 如能将成像仪与算法一体化, 可以研发 新的观测系统, 有助于提升城市等具有高度空间异质 性下垫面的测算精度, 并认识蒸散的三维分布规律.

蒸散研究涉及面广, 涉及参变量多, 如反照率、长 波辐射、短波辐射、温度等. 这些变量自身的物理型 尺度转换是实现蒸散的物理型尺度转换的必然前提. 因此, 从单一尺度到多尺度以及跨尺度之间, 需要开展 多变量、多仪器、多传感器、多学科交叉联合试验的 协作观测, 开展全球性协作, 从不同侧面、不同层面、 不同尺度上解析蒸散的变化过程, 并将各自具体的案 例解析, 努力上升到普适性理论构建. 例如, 目前有关 复杂下垫面的蒸散过程和参数关系研究仍然相对分 散, 尤其是稳定层结条件下湍流运动作用, 缺乏完整性 和系统性, 难以达成统一的参数化方案而在应用上受 到制约, 即使在国际上广泛应用的大气模式陆面参数 化方案中也鲜有体现. 针对蒸散测算的瓶颈问题开展 观测试验, 将为蒸散理论方法的突破等提供坚实的数 据基础, 有助于广泛地推动气象学、气候学、水文 学、生态学、环境科学以及水文气象和生态水文等交 叉学科的融合发展.

\section{5 结语}

作为地球水分循环与能量转换的关键环节, 蒸散 是边界层气象学、水文气象学和陆地水文学的重要研 究内容. 陆域蒸散的精准测算是地球物理、生物和环 境过程研究的共同瓶颈.

在勾勒经典理论方法的发展脉络的基础上，本文 梳理了相关理论方法的学科背景、基本假设及局限 
性, 总结了经典理论方法的制约瓶颈. 近三十年来, 随 着蒸散观测系统的不断普及, 新的观测事实和新的学 科理念的不断出现，新的理论也不断被探索. 固定实 验点位高频观测和航空航天遥感技术构筑了从植物气 孔到流域的跨尺度测算手段，为蒸散过程观测及机理 解析提供了纵向深化及横向综合的发展平台.

蒸散测算不确定性的主要来源, 首先, 蒸散测算不 确定性主要来源于对蒸散物理过程的认识不足，蒸散 广延性带来的时空异质性, 以及现有仪器观测能力的 局限性. 因此, 要在充分理解经典理论方法的基础上, 重视新观测现象所带来的启示，发展更能反映蒸散物 理本质的主导方程, 实现根本性突破. 其次, 要充分发 挥多尺度观测系统在度量非均匀下垫面尺度效应中的 作用, 发展物理型尺度转换函数, 发展非均匀下垫面适 用的蒸散理论方法. 最后, 在充分发挥现有观测系统的 同时, 仍要高度重视改进或研发新型观测系统, 针对理 论研究瓶颈, 开展精细试验设计与观测, 尤其是多学科 交叉的联合试验，为蒸散理论方法的整体突破等提供 坚实的数据基础.

陆域蒸散新理念、新模型、新技术的整体性发 展, 成为突破这一难题的现实条件, 将为揭示地球系统 的水-热-碳循环机理等全球性重大基础理论问题，为 解决灌溉农业与粮食安全、水资源高效利用与生态环 境保护、城市热环境调控与全球升温适应对策等新时 代国家重大需求等，提供更为严谨坚实的科学理论 支撑.

\section{参考文献}

高由禧. 1989. 关于我所开展干旱气候研究的历史. 高原气象，8: 103-106

刘元波, 吴桂平, 柯长青. 2016. 水文遥感. 北京: 科学出版社

朴世龙, 张新平, 陈安平, 刘强, 连旭, 王旭辉, 彭书时, 吴秀臣. 2019 . 极端气候事件对陆地生态系统碳循环的影响. 中国科学: 地球科 学, 49: 1321-1334

苏涛, 封国林. 2015. 基于不同再分析资料的全球蒸发量时空变化特 征分析. 中国科学: 地球科学, 45: 351-365

孙岗, 吴国雄. 2001. 陆面蒸散对气候变化的影响. 中国科学D辑: 地 球科学, 31: 59-69

Wang Jingfeng, 刘元波, 张珂. 2019. 最大熵增地表蒸散模型: 原理及 应用综述. 地球科学进展, 34: 596-605

王宁, 贾立, 李占胜, 李娜娜, 胡光成. 2016. 非参数化蒸散发估算方 法在黑河流域的适用性分析. 高原气象, 35: 118-128
于贵瑞, 张雷明, 孙晓敏. 2014. 中国陆地生态系统通量观测研究网 络(ChinaFLUX)的主要进展及发展展望. 地理科学进展, 33: 903917

张圆, 贾贞贞, 刘绍民, 徐自为, 徐同仁, 姚云军, 马燕飞, 宋立生, 李 相, 胡骁, 王泽宇, 郭枝虾, 周纪. 2020. 遥感估算地表蒸散发真实 性检验研究进展. 遥感学报, 24: 975-999

Abtew W, Melesse A. 2013. Evaporation and Evapotranspiration: Measurements and Estimations. Dordrecht: Springer Science and Business Media. 206

Ahiman O, Mekhmandarov Y, Pirkner M, Tanny J. 2018. Application of the flux-variance technique for evapotranspiration estimates in three types of agricultural structures. Int J Agronomy, 2018: 7935140

Anderson M C, Norman J M, Diak G R, Kustas W P, Mecikalski R J. 1997. A two-source time-integrated model for estimating surface fluxes using thermal infrared remote sensing. Remote Sens Environ, 60: $195-216$

Arnfield A J. 2003. Two decades of urban climate research: A review of turbulence, exchanges of energy and water, and the urban heat island. Int J Climatol, 23: 1-26

Aubinet M, Vesala T, Papale D. 2012. Eddy Covariance: A Practical Guide to Measurement and Data Analysis. Dordrecht, Heidelberg, London, New York: Springer Science and Business Media. 438

Baldocchi D. 2013. A brief history on eddy covariance flux measurements: A personal perspective. FluxLetter, 5: 1-8

Baldocchi D, Falge E, Gu L H, Olson R, Hollinger D, Running S, Anthoni P, Bernhofer C, Davis K, Evans R, Fuentes J, Goldstein A, Katul G, Law B, Lee X H, Malhi Y, Meyers T, Munger W, Oechel W, Paw K T, Pilegaard K, Schmid H P, Valentini R, Verma S, Vesala T, Wilson K, Wofsy S. 2001. FLUXNET: A new tool to study the temporal and spatial variability of ecosystem-scale carbon dioxide, water vapor, and energy flux densities. Bull Amer Meteorol Soc, 82: 2415-2434

Bastiaanssen W G M, Menenti M, Feddes R A, Holtslag A A M. 1998. A remote sensing surface energy balance algorithm for land (SEBAL). 1. Formulation. J Hydrol, 212-213: 198-212

Beyer M, Kühnhammer K, Dubbert M. 2020. In situ measurements of soil and plant water isotopes: A review of approaches, practical considerations and a vision for the future. Hydrol Earth Syst Sci, 24: 4413-4440

Blanken P D, Spence C, Hedstrom N, Lenters J D. 2011. Evaporation from Lake Superior: 1. Physical controls and processes. J Great Lakes Res, 37: 707-716

Bouchet R J. 1963. Evapotranspiration réelle evapotranspiration potentielle, signification climatique. Int Assoc Sci Hydrology, 62: 134-142 
Bowen I S. 1926. The ratio of heat losses by conduction and by evaporation from any water surface. Phys Rev, 27: 779-787

Bruce J P, Clark R H. 1966. Introduction to Hydrometeorology. Oxford: Pergamon Press Ltd.

Brügger N, Milligan I. 2019. The SAGE Handbook of Web History. London: SAGE Publications Ltd. 630

Brutsaert W. 1982. Evaporation into the Atmosphere: Theory, History, and Applications. Dordrecht: Springer Science and Business Media. 299

Brutsaert W. 2005. Hydrology: An Introduction. Cambridge: Cambridge University Press. 605

Brutsaert W. 2015. A generalized complementary principle with physical constraints for land-surface evaporation. Water Resour Res, 51: 8087-8093

Budyko M I. 1948. Evaporation under Natural Conditions. Leningrad: Gidrometeorizdat, 1963

Burgess S S O, Dawson T E. 2004. The contribution of fog to the water relations of Sequoia sempervirens (D. Don): Foliar uptake and prevention of dehydration. Plant Cell Environ, 27: 1023-1034

Burman R, Pochop L O. 1994. Evaporation, Evapotranspiration and Climatic Data. Amsterdam: Elsevier Science. 278

Caird M A, Richards J H, Donovan L A. 2007. Nighttime stomatal conductance and transpiration in $\mathrm{C} 3$ and C4 Plants. Plant Physiol, 143: $4-10$

Cermak J, Nadezhdina N. 1998. Sapwood as the scaling parameterdefining according to xylem water content or radial pattern of sap flow? Ann For Sci, 55: 509-521

Chen J W, Deng Y, Wang J F, Lin W S. 2017. Hindcasting the maddenjulian oscillation with a new parameterization of surface heat fluxes. J Adv Model Earth Syst, 9: 2696-2709

Cirelli D, Equiza M A, Lieffers V J, Tyree M T. 2016. Populus species from diverse habitats maintain high night-time conductance under drought. Tree Physiol, 36: 229-242

Clausnitzer F, Köstner B, Schwärzel K, Bernhofer C. 2011. Relationships between canopy transpiration, atmospheric conditions and soil water availability-Analyses of long-term sap-flow measurements in an old Norway spruce forest at the Ore Mountains/Germany. Agric For Meteorol, 151: 1023-1034

Crago R D. 1996. Conservation and variability of the evaporative fraction during the daytime. J Hydrol, 180: 173-194

Craig H, Gordon L I. 1965. Deuterium and oxygen-18 variations in the ocean and the marine atmosphere. In: Tongiorgi E, ed. Stable isotopes in Oceanographic Studies and Paleotemperatures. Spoleto: Cons. Naz. di Rech. 9-130

Cristea N C, Kampf S K, Burges S J. 2013. Revised coefficients for Priestley-Taylor and Makkink-Hansen equations for estimating daily reference evapotranspiration. J Hydrol Eng, 18: 1289-1300

Cui Y F, Liu Y B, Gan G J, Wang R N. 2020. Hysteresis behavior of surface water fluxes in a hydrologic transition of an ephemeral lake. J Geophys Res-Atmos, 125: e32364

Cuxart J, Boone A A. 2020. Evapotranspiration over land from a boundary-layer meteorology perspective. Bound-Layer Meteorol, 177: $427-459$

Daley M J, Phillips N G. 2006. Interspecific variation in nighttime transpiration and stomatal conductance in a mixed New England deciduous forest. Tree Physiol, 26: 411-419

Dalton J. 1802. Experimental essays on the constitution of mixed gases; on the force of steam or vapor from water and other liquids in different temperatures, both in a Torricellian vacuum and in air; on evaporation and on the expansion of gases by heat. Mem Manchester Lit and Phil Soc, 5: 535-602

de Bruin H A R. 1983. A model for the Priestley-Taylor parameter $\alpha$. J Clim Appl Meteor, 22: 572-578

de la Hire P. 1720. Mémoires de mathematique et de physique., tirés des registres de l'Academie royale des sciences, de l'année MDCCIII. Remarques sur l'eau de la pluie, sur l'origine des fontaines; avec quelques particularités sur la construction des citernes. Hist de l'Acad Roy des Sci Ann, 1703: 56-59

de Vries D A. 1959. The Influence of irrigation on the energy balance and the climate near the ground. J Meteor, 16: 256-270

Dyer A J. 1974. A review of flux-profile relationships. Bound-Layer Meteorol, 7: 363-372

Dye P J, Soko S, Poulter A G. 1996. Evaluation of the heat pulse velocity method for measuring sap flow in Pinus patula. J Exp Bot, 47: $975-981$

Eller C B, Lima A L, Oliveira R S. 2013. Foliar uptake of fog water and transport belowground alleviates drought effects in the cloud forest tree species, Drimys brasiliensis (Winteraceae). New Phytol, 199: $151-162$

Engelmann C, Bernhofer C. 2016. Exploring eddy-covariance measurements using a spatial approach: The eddy matrix. Bound-Layer Meteorol, 161: 1-17

Fisher J B, Melton F, Middleton E, Hain C, Anderson M, Allen R, McCabe M F, Hook S, Baldocchi D, Townsend P A, Kilic A, Tu K, Miralles D D, Perret J, Lagouarde J P, Waliser D, Purdy A J, French A, Schimel D, Famiglietti J S, Stephens G, Wood E F. 2017. The future of evapotranspiration: Global requirements for ecosystem functioning, carbon and climate feedbacks, agricultural management, and water resources. Water Resour Res, 53: 2618-2626

Foken T. 2008. Micrometeorology. Berlin, Heidelberg: Springer-Verlag. 306

Foken T, Aubinet M, Leuning R. 2012. The Eddy Covariance Method. 
In: Eddy Covariance: A Practical Guide to Measurement and Data Analysis. Dordrecht, Heidelberg, London, New York: Springer Science and Business Media. 438

Gan G J, Liu Y B, Sun G. 2021. Understanding interactions among climate, water, and vegetation with the Budyko framework. EarthSci Rev, 212: 103451

Gianniou S K, Antonopoulos V Z. 2007. Evaporation and energy budget in Lake Vegoritis, Greece. J Hydrol, 345: 212-223

Glenn E P, Nagler P L, Huete A R. 2010. Vegetation index methods for estimating evapotranspiration by remote sensing. Surv Geophys, 31: $531-555$

Grachev A A, Andreas E L, Fairall C W, Guest P S, Persson P O G. 2015. Similarity theory based on the Dougherty-Ozmidov length scale. Quart J R Meteorol Soc, 141: 1845-1856

Granier A. 1987. Evaluation of transpiration in a Douglas-fir stand by means of sap flow measurements. Tree Physiol, 3: 309-320

Guo Y H, Zhang Y S, Ma N, Xu J Q, Zhang T. 2019. Long-term changes in evaporation over Siling Co Lake on the Tibetan Plateau and its impact on recent rapid lake expansion. Atmos Res, 216: 141-150

Han S J, Tian F Q. 2020. A review of the complementary principle of evaporation: From the original linear relationship to generalized nonlinear functions. Hydrol Earth Syst Sci, 24: 2269-2285

Haughton N, Abramowitz G, Pitman A J, Or D, Best M J, Johnson H R, Balsamo G, Boone A, Cuntz M, Decharme B, Dirmeyer P A, Dong J, Ek M, Guo Z, Haverd V, van den Hurk B J J, Nearing G S, Pak B, Santanello Jr J A, Stevens L E, Vuichard N. 2016. The plumbing of land surface models: Is poor performance a result of methodology or data quality? J Hydrometeorol, 17: 1705-1723

Higbie R. 1935. The rate of absorption of a pure gas into a still liquid during short periods of exposure. Trans AIChE, 31: 365-388

Horst T W, Weil J C. 1994. How far is far enough? The fetch requirements for micrometeorological measurement of surface fluxes. J Atmos Ocean Technol, 11: 1018-1025

Howell T A, Schneider A D, Dusek D A, Marek T H, Steiner J L. 1995 Calibration and scale performance of Bushland weighing lysimeters. Trans ASAE, 38: 1019-1024

Hu Y G, Buttar N A, Tanny J, Snyder R L, Savage M J, Lakhiar I A. 2018. Surface renewal application for estimating evapotranspiration: A review. Adv Meteor, 2018: 1-11

Huber B. 1932. Observation and measurements of sap flow in plant. Ber Dtsch Bot Ges, 50: 89-109

Itier B, Brunet Y, McAneney K J, Lagouarde J P. 1994. Downwind evolution of scalar fluxes and surface resistance under conditions of local advection. Part I: A reappraisal of boundary conditions. Agric For Meteorol, 71: 211-225

Jackson R D. 1985. Evaluating evapotranspiration at local and regional scales. Proc IEEE, 73: 1086-1096

Jacobs A F G, Heusinkveld B G, Wichink Kruit R J, Berkowicz S M. 2006. Contribution of dew to the water budget of a grassland area in the Netherlands. Water Resour Res, 42: W03415

Jiang L, Islam S. 1999. A methodology for estimation of surface evapotranspiration over large areas using remote sensing observations. Geophys Res Lett, 26: 2773-2776

Keeling C D. 1958. The concentration and isotopic abundances of atmospheric carbon dioxide in rural areas. Geochim Cosmochim Acta, 13: 322-334

Khalil M, Sakai M, Mizoguchi M, Miyazaki T. 2003. Current and prospective applications of zero flux plane (ZFP) method. J Jpn Soc Soil Phys, 95: 75-90

Kidron G J, Starinsky A, Yaalon D H. 2014. Cyanobacteria are confined to dewless habitats within a dew desert: Implications for past and future climate change for lithic microorganisms. J Hydrol, 519: 3606-3614

Leclerc M, Foken T. 2014. Footprints in Micrometeorology and Ecology. Berlin, Heidelberg: Springer. 239

Lee X H. 2018. Fundamentals of Boundary-Layer Meteorology. Gewerbestrasse: Springer International Publishing. 256

Lemone M A, Angevine W M, Bretherton C S, Chen F, Dudhia J, Fedorovich E, Katsaros K B, Lenschow D H, Mahrt L, Patton E G, Sun J, Tjernström M, Weil J. 2018. 100 years of progress in boundary layer meteorology. Meteorol Monogr, 59: 1-85

Lewis G N, Cornish R E. 1933. Separation of the isotopic forms of water by fractional distillation. J Am Chem Soc, 55: 2616-2617

Lewis J M. 1995. The story behind the Bowen ratio. Bull Amer Meteorol Soc, 76: 2433-2443

Li X Y, Ma Y J, Huang Y M, Hu X, Wu X C, Wang P, Li G Y, Zhang S Y, Wu H W, Jiang Z Y, Cui B L, Liu L. 2016. Evaporation and surface energy budget over the largest high-altitude saline lake on the Qinghai-Tibet Plateau. J Geophys Res-Atmos, 121: 1047010485

Li Z L, Tang R, Wan Z M, Bi Y Y, Zhou C, Tang B H, Yan G J, Zhang X Y. 2009. A review of current methodologies for regional evapotranspiration estimation from remotely sensed data. Sensors, 9: $3801-3853$

Liu S M, Li X, Xu Z W, Che T, Xiao Q, Ma M G, Liu Q H, Jin R, Guo J W, Wang L X, Wang W Z, Qi Y, Li H Y, Xu T R, Ran Y H, Hu X L, Shi S J, Zhu Z L, Tan J L, Zhang Y, Ren Z G. 2018. The Heihe integrated observatory network: A basin-scale land surface processes observatory in China. Vadose Zone J, 17: 180072

Liu S M, Xu Z W. 2019. Micrometeorological methods to determine evapotranspiration. In: Li X, Vereecken L, eds. Observation and Measurement of Ecohydrological Processes. Berlin, Heidelberg: 
Springer-Verlag Press

Liu S M, Xu Z W, Song L S, Zhao Q Y, Ge Y, Xu T R, Ma Y F, Zhu Z

L, Jia Z Z. 2016. Upscaling evapotranspiration measurements from multi-site to the satellite pixel scale over heterogeneous land surfaces. Agric For Meteorol, 230-231: 97-113

Liu S M, Xu Z W, Wang W Z, Jia Z Z, Zhu M J, Bai J, Wang J M. 2011. A comparison of eddy-covariance and large aperture scintillometer measurements with respect to the energy balance closure problem. Hydrol Earth Syst Sci, 15: 1291-1306

Liu Y B, Hiyama T. 2007. Detectability of day-to-day variability in the evaporative flux ratio: A field examination in the Loess Plateau of China. Water Resour Res, 43: W08503

Liu Y B, Hiyama T, Yamaguchi Y. 2006. Scaling of land surface temperature using satellite data: A case examination on ASTER and MODIS products over a heterogeneous terrain area. Remote Sens Environ, 105: 115-128

Liu Y B, Hiyama T, Yasunari T, Tanaka H. 2012. A nonparametric approach to estimating terrestrial evaporation: Validation in eddy covariance sites. Agric For Meteorol, 157: 49-59

Ma N, Szilagyi J. 2019. The CR of evaporation: A calibration-free diagnostic and benchmarking tool for large-scale terrestrial evapotranspiration modeling. Water Resour Res, 55: 7246-7274

Mahrt L. 1999. Stratified atmospheric boundary layers. Bound-Layer Meteor, 90: 375-396

Mahrt L. 2007. Weak-wind mesoscale meandering in the nocturnal boundary layer. Environ Fluid Mech, 7: 331-347

Mahrt L. 2010. Variability and maintenance of turbulence in the very stable boundary layer. Bound-Layer Meteorol, 135: 1-18

Manley G. 1958. On the frequency of snowfall in metropolitan England. Quart J R Met Soc, 84: 70-72

Marshall D C. 1958. Measurement of sap flow in conifers by heat transport. Plant Physiol, 33: 385-396

McNaughton K G. 1976. Evaporation and advection I: Evaporation from extensive homogeneous surfaces. Quart J R Met Soc, 102: 181-191

Moene A F, Beyrich F, Hartogensis O K. 2009. Developments in scintillometry. Bull Amer Meteorol Soc, 90: 694-698

Monin A, Obukhov A. 1954. Basic turbulence mixing laws in the atmospheric surface layer. Trudy Geologicheskogo Instituta Akademiya Nauk, Svaz Sovetskych Socialistickych Republik, 24: 163187

Monteith J L. 1965. Evaporation and environment. Symp Soc Exp Biol, 19: $205-234$

Montgomery R B. 1948. Vertical eddy flux of heat in the atmosphere. J Meteor, 5: 265-274

Moore G W, Cleverly J R, Owens M K. 2008. Nocturnal transpiration in riparian Tamarix thickets authenticated by sap flux, eddy covariance and leaf gas exchange measurements. Tree Physiol, 28: $521-528$

Norman J M, Kustas W P, Humes K S. 1995. Source approach for estimating soil and vegetation energy fluxes in observations of directional radiometric surface temperature. Agric For Meteorol, 77: 263-293

Nouri H, Nagler P, Chavoshi Borujeni S, Barreto Munez A, Alaghmand S, Noori B, Galindo A, Didan K. 2020. Effect of spatial resolution of satellite images on estimating the greenness and evapotranspiration of urban green spaces. Hydrological Processes, 34: 3183-3199

Novák V. 2012. Evapotranspiration in the Soil-Plant-Atmosphere System. Dordrecht: Springer

Ogle K, Lucas R W, Bentley L P, Cable J M, Barron-Gafford G A, Griffith A, Ignace D, Jenerette G D, Tyler A, Huxman T E, Loik M E, Smith S D, Tissue D T. 2012. Differential daytime and night-time stomatal behavior in plants from North American deserts. New Phytol, 194: 464-476

Oki T, Kanae S. 2006. Global hydrological cycles and world water resources. Science, 313: 1068-1072

Pan X, Liu Y B, Gan G J, Fan X W, Yang Y B. 2017. Estimation of evapotranspiration using a nonparametric approach under all sky: Accuracy evaluation and error analysis. IEEE J Sel Top Appl Earth Observations Remote Sens, 10: 2528-2539

Pan X, You C S, Liu Y B, Shi C X, Han S, Yang Y B, Hu J. 2020. Evaluation of satellite-retrieved evapotranspiration based on a nonparametric approach over an arid region. Int J Remote Sens, 41: 7605-7623

Paw U K T, Brunet Y, Collineau S, Shaw R H, Maitani T, Qiu J, Hipps L. 1992. On coherent structures in turbulence above and within agricultural plant canopies. Agric For Meteorol, 61: 55-68

Peixoto J P. 1995. The role of the atmosphere in the water cycle. The Role of Water and the Hydrological Cycle in Global Change. Berlin, Heidelberg: Springer. 199-252

Penman H L. 1948. Natural evaporation from open water, bare soil and grass. Proc R Soc Lond A, 193: 120-145

Philip J R. 1959. The theory of local advection: I. J Meteor, 16: 535547

Phillips D L, Gregg J W. 2001. Uncertainty in source partitioning using stable isotopes. Oecologia, 127: 171-179

Pitman A J. 2003. The evolution of, and revolution in, land surface schemes designed for climate models. Int J Climatol, 23: 479-510

Prandtl L. 1905. Über Flüssigkeitsbewegung bei sehr kleiner Reibung. Verhaglungen des Dritten Internationalen Mathematiker-Kongresses in Heidelberg 1904. Krazer A, ed. Leipzig: Teubner. 484-491

Price J C. 1980. The potential of remotely sensed thermal infrared data 
to infer surface soil moisture and evaporation. Water Resour Res, 16: $787-795$

Priestley C H B, Taylor R J. 1972. On the assessment of surface heat flux and evaporation using large-scale parameters. Mon Weather Rev, 100: 81-92

Qiu G Y. 1996. A new method for estimation of evapotranspiration. Dissertation for Doctoral Degree. Tottori: Tottori University, United Graduate School of Agriculture Science

Qiu G Y, Tan S L, Wang Y, Yu X H, Yan C H. 2017. Characteristics of evapotranspiration of urban lawns in a sub-tropical megacity and its measurement by the "Three Temperature Model + Infrared Remote Sensing" method. Remote Sens, 9: 502

Qiu G Y, Yano T, Momii K. 1998. An improved methodology to measure evaporation from bare soil based on comparison of surface temperature with a dry soil surface. J Hydrol, 210: 93-105

Qiu G Y, Yu X H, Wen H Y, Yan C H. 2020. An advanced approach for measuring the transpiration rate of individual urban trees by the $3 \mathrm{D}$ three-temperature model and thermal infrared remote sensing. J Hydrol, 587: 125034

Rao B Q, Liu Y D, Wang W B, Hu C X, Li D H, Lan S B. 2009. Influence of dew on biomass and photosystem II activity of cyanobacterial crusts in the Hopq Desert, northwest China. Soil Biol Biochem, 41: 2387-2393

Ren Y, Zhang H S, Wei W, Wu B G, Cai X H, Song Y. 2019. Effects of turbulence structure and urbanization on the heavy haze pollution process. Atmos Chem Phys, 19: 1041-1057

Reynolds O. 1895. On the dynamical theory of incompressible viscous fluids and the determination of the criterion. Phil Trans R Soc Lond A $186: 123-161$

Richards L A. 1954. Multiple tensiometer for determining the vertical component of the hydraulic gradient in soill. Soil Sci Soc Am J, 18: $7-10$

Roderick M L, Farquhar G D. 2002. The cause of decreased pan evaporation over the past 50 years. Science, 298: 1410-1411

Chabot R, Bouarfa S, Zimmer D, Chaumont C, Moreau S. 2005. Evaluation of the sap flow determined with a heat balance method to measure the transpiration of a sugarcane canopy. Agric Water Manage, 75: 10-24

Rossby C G. 1932. A generalization of the theory of the mixing length with applications to oceanic and atmospheric turbulence. Mass Inst Tech Meteorl Pap, 1: 1-36

Schmidt W. 1915. Strahlung und Verdunstung an freien Wasserfliichen: ein Beitrag zum Wiirmehaushalt des Weltmeers und zum Wasserhaushalt der Erde. Ann d Hydrogr u Mar Met, 43: 111-124

Schmugge T J, André J C. 1991. Land Surface Evaporation. New York: Springer. 424
Sellers P J, Hall F G, Kelly R D, Black A, Baldocchi D, Berry J, Ryan M, Jon Ranson K, Crill P M, Lettenmaier D P, Margolis H, Cihlar J, Newcomer J, Fitzjarrald D, Jarvis P G, Gower S T, Halliwell D, Williams D, Goodison B, Wickland D E, Guertin F E. 1997. BOREAS in 1997: Experiment overview, scientific results, and future directions. J Geophys Res, 102: 28731-28769

Shuttleworth W J. 2007. Putting the "vap" into evaporation. Hydrol Earth Syst Sci, 11: 210-244

Shuttleworth W J. 2012. Terrestrial Hydrometeorology. West Sussex: John Wiley \& Sons, Ltd

Shuttleworth W J, Wallace J S. 1985. Evaporation from sparse crops-an energy combination theory. Quart J R Meteorol Soc, 111: 839-855

Spronken-Smith R A, Oke T R, Lowry W P. 2000. Advection and the surface energy balance across an irrigated urban park. Int J Climatol, 20: $1033-1047$

Stammers W N, Igwe O C, Whiteley H R. 1973. Calculation of evaporation from measurements of soil water and the soil water characteristics. Can Agr Eng, 15: 2-5

Su Z. 2002. The surface energy balance system (SEBS) for estimation of turbulent heat fluxes. Hydrol Earth Syst Sci, 6: 85-100

Sun J, Lenschow D H, Burns S P, Banta R M, Newsom R K, Coulter R, Frasier S, Ince T, Nappo C, Balsley B B, Jensen M, Mahrt L, Miller D, Skelly B. 2004. Atmospheric disturbances that generate intermittent turbulence in nocturnal boundary layers. Bound-Layer Meteor, 110: 255-279

Swanson R H, Whitfield D W A. 1981. A numerical analysis of heat pulse velocity theory and practice. J Exp Bot, 32: 221-239

Swinbank W C. 1951. The measurement of vertical transfer of heat and water vapor by eddies in the lower atmosphere. J Meteor, 8: 135-145

Taha H, Akbari H, Rosenfeld A. 1991. Heat island and oasis effects of vegetative canopies: Micro-meteorological field-measurements. Theor Appl Climatol, 44: 123-138

Tanner C B. 1960. Energy balance approach to evapotranspiration from crops. Soil Sci Soc Am J, 24: 1-9

Tatarskii V I. 1961. Wave Propagation in a Turbulent Medium. New York: McGraw-Hill. 285

Taylor G I. 1938. The spectrum of turbulence. Proc R Soc Lond A, 164: 476-490

Thornthwaite C W, Holzman B. 1942. Measurement of evaporation from land and water surfaces. Technical Bulletin. Washington: United States Department of Agriculture

Tillman J E. 1972. The indirect determination of stability, heat and momentum fluxes in the atmospheric boundary layer from simple scalar variables during dry unstable conditions. J Appl Meteorol, 11: 783-792

Todd R W, Evett S R, Howell T A. 2000. The Bowen ratio-energy 
balance method for estimating latent heat flux of irrigated alfalfa evaluated in a semi-arid, advective environment. Agric For Meteorol, 103: 335-348

Uclés O, Villagarcía L, Moro M J, Canton Y, Domingo F. 2014. Role of dewfall in the water balance of a semiarid coastal steppe ecosystem. Hydrol Process, 28: 2271-2280

von Kármán T. 1930. Mechanische Ähnlichkeit und Turbulenz, Nachrichten von der Gesellschaft der Wissenschaften ZU Göttingen. Mathematisch-Physikalische Klasse, 58-76

Walker C D, Richardson S B. 1991. The use of stable isotopes of water in characterising the source of water in vegetation. Chem Geol, 94: $145-158$

Wang J F, Bras R L. 2011. A model of evapotranspiration based on the theory of maximum entropy production. Water Resour Res, 47: W03521

Wang J F, Bras R L, Lerdau M, Salvucci G D. 2007. A maximum hypothesis of transpiration. J Geophys Res, 112: G03010

Wang J F, Salvucci G D, Bras R L. 2004. An extremum principle of evaporation. Water Resour Res, 40: W09303

Wang K, Dickinson R E. 2012. A review of global terrestrial evapotranspiration: Observation, modeling, climatology, and climatic variability. Rev Geophys, 50: RG2005

Wang W, Xiao W, Cao C, Gao Z Q, Hu Z H, Liu S D, Shen S H, Wang L L, Xiao Q T, Xu J P, Yang D, Lee X H. 2014. Temporal and spatial variations in radiation and energy balance across a large freshwater lake in China. J Hydrol, 511: 811-824

Ward R C, Robinson M. 2000. Principles of Hydrology. 4th ed. London: McGraw-Hill

Wei W, Zhang H S, Wu B G, Huang Y X, Cai X H, Song Y, Li J D. 2018. Intermittent turbulence contributes to vertical dispersion of $\mathrm{PM}_{2.5}$ in the North China Plain: Cases from Tianjin. Atmos Chem Phys, 18: 12953-12967

Wesely M L. 1976. The combined effect of temperature and humidity fluctuations on refractive index. J Appl Meteorol, 15: 43-49

Willis W O. 1960. Evaporation from layered soils in the presence of a water table. Soil Sci Soc Am J, 24: 239-242

World Meteorological Organization (WMO). 1996. Guide to Meteorological Instruments and Methods of Observation. WMO-No. 8, 6th ed. Geneva: World Meteorological Organization

Wu J G, Jelinski D E, Luck M, Tueller P T. 2000. Multiscale analysis of landscape heterogeneity: Scale variance and pattern metrics. Geogr Inf Sci, 6: 6-19

Xiong Y J, Zhao W L, Wang P, Paw U K T, Qiu G Y. 2019. Simple and applicable method for estimating evapotranspiration and its components in arid regions. J Geophys Res-Atmos, 124: 9963-9982

Yakir D, Sternberg L S L. 2000. The use of stable isotopes to study ecosystem gas exchange. Oecologia, 123: 297-311

Yakir D, Wang X F. 1996. Fluxes of $\mathrm{CO}_{2}$ and water between terrestrial vegetation and the atmosphere estimated from isotope measurements. Nature, 380: 515-517

Yan C H, Qiu G Y. 2016. The three-temperature model to estimate evapotranspiration and its partitioning at multiple scales: A review. Trans ASABE, 59: 661-670

Yu M H, Ding G D, Gao G L, Zhao Y Y, Sai K. 2019. Hysteresis resulting in forestry heat storage underestimation: A case study of plantation forestry in northern China. Sci Total Environ, 671: 608-616

Zeppel M J B, Lewis J D, Phillips N G, Tissue D T. 2014. Consequences of nocturnal water loss: A synthesis of regulating factors and implications for capacitance, embolism and use in models. Tree Physiol, 34: 1047-1055

Zeweldi D A, Gebremichael M, Wang J M, Sammis T, Kleissl J, Miller D. 2010. Intercomparison of sensible heat flux from large aperture scintillometer and eddy covariance methods: Field experiment over a homogeneous semi-arid region. Bound-Layer Meteorol, 135: 151-159

Zhang K, Kimball J S, Nemani R R, Running S W. 2010. A continuous satellite-derived global record of land surface evapotranspiration from 1983 to 2006. Water Resour Res, 46: W09522

Zhang L, Dawes W R, Walker G R. 2001. Response of mean annual evapotranspiration to vegetation changes at catchment scale. Water Resour Res, 37: 701-708

Zhao W L, Gentine P, Reichstein M, Zhang Y, Zhou S, Wen Y Q, Lin C J, Li X, Qiu G Y. 2019. Physics-constrained machine learning of evapotranspiration. Geophys Res Lett, 46: 14496-14507

Zhao X S, Liu Y B. 2017. Phase transition of surface energy exchange in China's largest freshwater lake. Agric For Meteorol, 244-245: 98-110

Zhao X S, Liu Y B. 2018. Variability of surface heat fluxes and its driving forces at different time scales over a large ephemeral lake in China. J Geophys Res-Atmos, 123: 4939-4957

Zhao X S, Liu Y B, Tanaka H, Hiyama T. 2010. A comparison of flux variance and surface renewal methods with eddy covariance. IEEE $\mathrm{J}$ Sel Top Appl Earth Observations Remote Sens, 3: 345-350

Zhuang Y L, Zhao W Z. 2017. Dew formation and its variation in Haloxylon ammodendron plantations at the edge of a desert oasis, northwestern China. Agric For Meteorol, 247: 541-550

Zou Z D, Yang Y J, Qiu G Y. 2019. Quantifying the evapotranspiration rate and its cooling effects of urban hedges based on three-temperature model and infrared remote sensing. Remote Sens, 11: 202

(责任编委: 施建成) 